\title{
In vivo Multi-scale Photoacoustic Imaging Guided Photothermal Therapy of Cervical Cancer based on Customized Laser System and Targeted Nanoparticles
}

This article was published in the following Dove Press journal:

International Journal of Nanomedicine

\section{Ting Qiu' ${ }^{1} *$ \\ Yintao Lan ${ }^{1} *$ \\ Zuwu Wei ${ }^{2}$ \\ Yanfen Zhang ${ }^{3}$ \\ Yanping Lin' \\ Chenggong $\mathrm{Tu}^{\prime}$ \\ Guangjuan Mao' \\ Lingmin Zhang ${ }^{3}$ \\ Bin Yang' \\ Jian Zhang $\mathbb{D}^{\prime}$}

'The Sixth Affiliated Hospital of Guangzhou Medical University, Qingyuan People's Hospital, Department of Biomedical Engineering, School of Basic Medical Sciences, Guangzhou Medical University, Guangzhou, 5I I436, People's Republic of China; ${ }^{2}$ The United Innovation of Mengchao Hepatobiliary Technology Key Laboratory of Fujian Province, Mengchao Hepatobiliary Hospital of Fujian Medical University, Fujian, 350025, People's Republic of China; ${ }^{3}$ School of Pharmaceutical Sciences, Guangzhou Medical University, Guangzhou, 5I I 436, Guangdong, People's Republic of China

*These authors contributed equally to this work
Background: Effective treatment strategy for cervical carcinoma is subject to the limitation of its anatomical location and histological characteristics. Comprehensive imaging before cervical carcinoma treatment is of great significance for the patients. Current imaging methods cannot meet the requirements of high resolution, deep imaging depth and noninvasive imaging at the same time. Fortunately, Photoacoustic imaging (PAI) is a novel imaging method that combines rich optical contrast, high ultrasonic spatial resolution, and deep penetration depth in a single modality. Moreover, PAI-guided photothermal therapy (PTT) by aid of targeting nanoparticles is an emerging and effective cancer treatment in recent years.

Methods: Here, strong near-infrared region (NIR) absorption-conjugated polymer PIIGDTS (PD) nanoparticles with folic acid (FA) modification (namely, PD-FA) that targeted at Hela cell were specifically designed as cervical tumor imaging contrast agents and photothermal agents.

Results: The obtained PD-FA nanoparticles exhibited admirable photoacoustic contrastenhancing ability and desirable PTT behavior with the photothermal conversion efficiency as high as $62.6 \%$ in vitro. Furthermore, the PAI performance and PTT efficiency were tested in HeLa tumor-bearing nude mice after injection of PD-FA nanoparticles. In vivo multiscale, PAI provided B-san and 3D dimension imaging for intuitive and comprehensive information of Hela tumor. Moreover, the Hela tumor can be completely eliminated within 18 days after PTT, with no toxicity and side effects.

Conclusion: In summary, PD-FA injection combined with PAI and PTT systems provides a novel powerful tool for early diagnosis and precise treatment of cervical cancer.

Keywords: cervical cancer, multi-scale photoacoustic imaging, photothermal therapy, PIIGDTS nanoparticle

\section{Introduction}

Cervical cancer is the fourth most common cancer among women and the second most frequent cause of cancer-related death worldwide, accounting for nearly 300,000 deaths annually. ${ }^{1-3}$ what's worse, the incidence of cervical cancer has been increasing with the steady increase in the infection rate of human papillomavirus (HPV) in past years. $^{4-7}$ Early detection and precise treatment are essential for the patients with cervical cancer. To decrease the unnecessary treatment and avoid recrudescence for 
the best treatment effect, a rational treatment program should be based on comprehensive imaging of tumor. Comprehensive tumor imaging could distinguish tumor and surrounding tissues or organs or the regional lymph nodes to confirm the clinical stage. Although current imaging methods including US, CT, MRI have been proved to be effective in cervical cancer staging, these methods have their own disadvantages and cannot meet the requirements of high resolution and deep imaging depth simultaneously. Moreover, subsequent histological analysis of tumor tissue is time-consuming and complicated. In addition, tumor tissue biopsy is invasive to human being. Therefore, the development of a new imaging method to accurately reveal further information about tumor without damaging the tissue will help the precise tumor therapy with improved effects and minimize complications.

As an emerging optical imaging strategy, photoacoustic imaging (PAI) is based on the photoacoustic effect, can sense acoustic pressure waves generated from absorbed photon energy. ${ }^{8-11}$ PAI ultrasonically overcomes the optical diffusion limit. ${ }^{12,13}$ Then, PAI combines rich optical contrasts with high ultrasonic spatial resolution. ${ }^{14-17}$ Currently, photoacoustic system has three major implementations: photoacoustic microscopy (PAM), photoacoustic computed tomography (PACT), and photoacoustic endoscopy (PAE). The PAM is designed for high-resolution $(1-50 \mu \mathrm{m})$ imaging over a shallow depth, while PACT is targeted at deeper imaging depth with a compromised spatial resolution $(50-200 \mu \mathrm{m}){ }^{18}$ Therefore, PAM can monitor single organelles and cells such as melanosomes, ${ }^{19}$ red blood cells (RBCs), ${ }^{20}$ and nucleic acids $^{21}$ in real-time and track subtle functional changes of disease microenvironments, including angiogenesis in the tumor, oxyhemoglobin $\left(\mathrm{HbO}_{2}\right)$ and deoxyhemoglobin $(\mathrm{Hb})$ in blood flow and lipid accumulation in atherosclerosis. $^{22-24}$ PACT allows brain, organ, and whole-body imaging from small animals to primates. $^{25-27}$ PAE can provide structural information of the esophagus and gastrointestinal tract. ${ }^{28}$ Moreover, multidimensional photoacoustic images can be obtained by aid of specific algorithms. ${ }^{29}$ Based on above characteristics, PAI is expected to be a promising application in biological medicine to circumvent the drawbacks of traditional imaging methods discussed above, such as the ionizing radiation, high cost, extended time requirements. In recent studies, PAI traditionally uses NIR as the optical window to acquire the maximum penetration depth for solid tumors and minimum damage to surrounding healthy tissues. $^{30,31}$ In this regard, PAI is a noninvasive imaging technique that can unveil comprehensive information of cervical cancer.

To date, photothermal therapy (PTT), which converts light energy into thermal energy to ablate tumors with the aid of photothermal agents, has attracted tremendous attention and emerged as a prospective method in the precise treatment of cancers. ${ }^{32-36}$ The greatest appeal of PTT in the field of cancer therapy is related to its specific selectivity to cancer-targeting sites and minimal invasion to the surrounding normal tissues. This precise and effective treatment strategy to deal with cancer can potentially break though the limit of traditional cancer therapies include chemotherapy, radiotherapy, and surgery, in which the patients may suffer from serious side effects and unsatisfactory treatment outcomes. ${ }^{37-39}$ Besides, extensive employment of nanomaterials with high photothermal conversion efficiency and excellent biocompatibility could greatly enhance the photothermal conversion efficiency and also increase the accumulation of photoacoustic agents in tumors. ${ }^{40}$

Currently, the combination of PAI and PTT has been widely applied in tumor therapy, but the exploration for cervical cancer treatment is not enough. ${ }^{41-47}$ Besides, it has a greater prospect that developed PAE would be applied in the treatment of cervical cancer as an intracavitary tumor. Furthermore, we designed a PIIGDTSbased nanoparticle (namely, PD nanoparticles) interacted with folic acid (FA) that specifically targets cervical cancer (namely, PD-FA nanoparticles). As illustrated in Figure 1, the PD-FA nanoparticle could specifically aggregate in tumor regions through enhanced permeability and retention (EPR) effects and ligand-receptor interactions. ${ }^{48-51}$ Extensive characterizations and experiments have been conducted to demonstrate the uniformly particle diameter, outstanding biocompatibility, good thermal conversion efficiency and excellent tumor ablation efficiency of PD-FA nanoparticles both in vitro and in vivo. Given all that, PAI-guided PTT by aid of PD-FA nanoparticles as therapeutic agents is a promising efficient and targeted treatment for cervical cancer.

\section{Materials and Methods}

\section{Materials}

3-(4,5-Dimethylthiazol-2-yl)-2,5-diphenyltetrazolium bromide (MTT) was purchased from Shanghai SigmaAldrich Biotechnology Co. Ltd. A LIVE/DEAD 


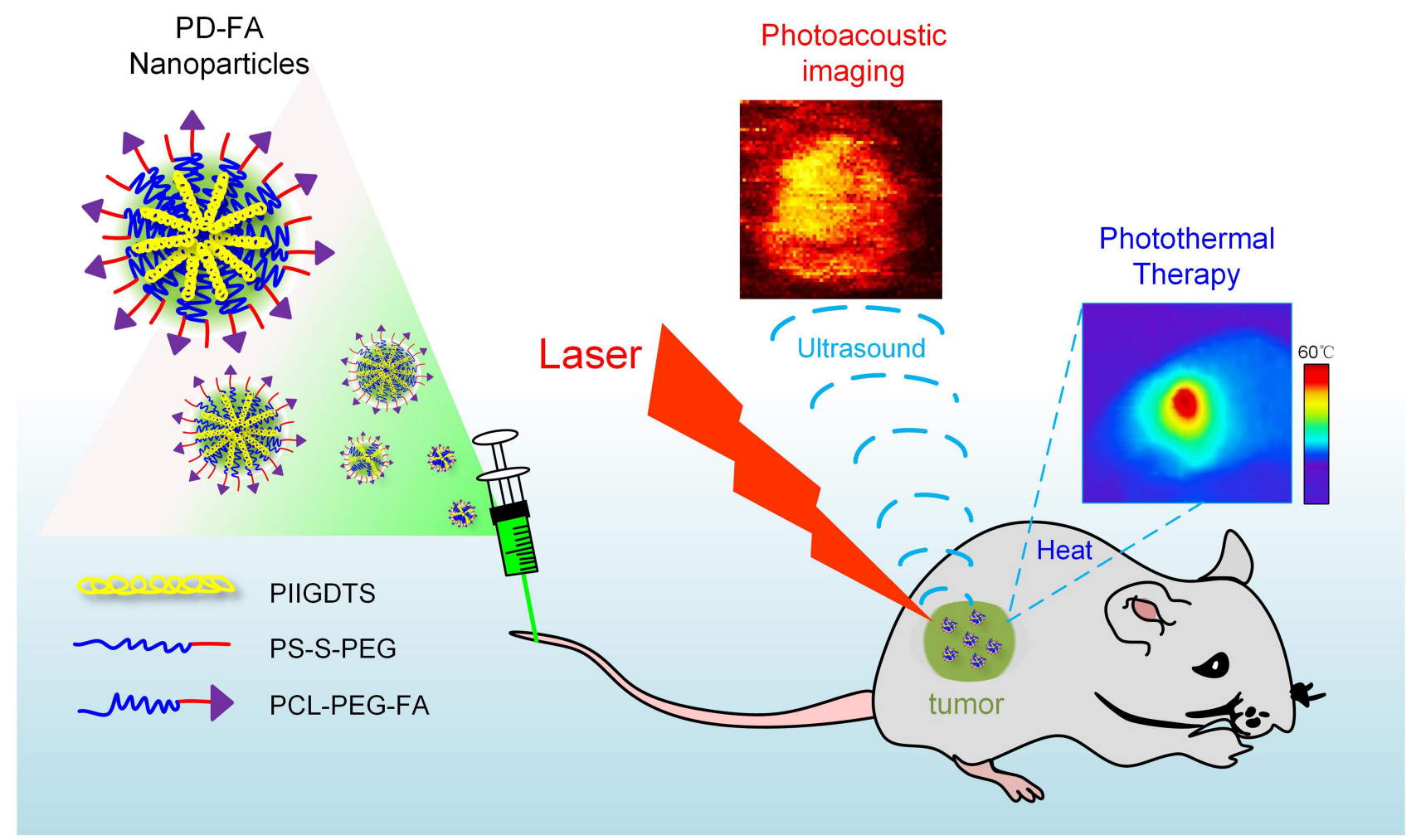

Figure I Schematic illustration of the photoacoustic imaging (PAI) guided photothermal therapy (PTT) with the assistance of PD-FA nanoparticles for the imaging and therapy of cervical carcinoma.

Viability/Cytotoxicity Kit was purchased from Shanghai Yesen Biotechnology Co. Ltd. Bovine serum albumin (BSA) was purchased from Guangzhou Nice Biotechnology Co. Ltd. All commercial reagents were used as received unless otherwise stated. Ultrapure water $\left(18.25 \mathrm{M} \Omega \cdot \mathrm{cm}, 25^{\circ} \mathrm{C}\right)$ was used in the experiments. All the reactions were carried out under an argon atmosphere.

\section{Characterization}

${ }^{1}$ HNMR spectra were recorded on a Bruker Ultrashield 500 Plus NMR spectrometer. The optical absorption spectrum of the nanoparticles was measured by a SHIMADZU UV-2600 spectrophotometer. Dynamic light scattering (DLS) was carried out by a Malvern Nano-ZS Particle Sizer. Transmission electron microscopy (TEM) image was conducted using a JEM-1400 plus transmission microscope. Laser-confocal scanning imaging was performed by a Zeiss LSM780 confocal microscope. Photoacoustic characterization was conducted with a homemade multi-spectral photoacoustic microscopy (MSPAM) system.

\section{Synthesis of PIIGDTS}

The semiconducting polymer was synthesized according to our previously reported method. ${ }^{52}$ (Scheme S1). (E)-2,2'Dibromo-4,4'-bis(2-decyltetradecyl)-[6,6'-bithieno[3,2-b] pyrrolylidene]-5,5'(4H,4'H)-dione (145 $\mathrm{mg}, 0.13 \mathrm{mmol}$ ), 4,4'-Bis(octyl)-5, 5'-bis (trimethyltin)-dithieno[3,2-b:2',3'd] silole (98 $\mathrm{mg}, 0.13 \mathrm{mmol}), \mathrm{Pd}_{2}(\mathrm{DBA})_{3}(8.4 \mathrm{mg})$, and $\mathrm{P}(\text { tolyl })_{3}(32 \mathrm{mg})$ were dissolved in $10 \mathrm{~mL}$ toluene and then refluxed for 48 hours. After cooling to room temperature, $80 \mathrm{~mL}$ of $\mathrm{CH}_{3} \mathrm{OH}$ was added, and the dark-green precipitate was collected by filtration. Then, the precipitate was purified with Soxhlet extraction using $\mathrm{CH}_{3} \mathrm{OH}, \mathrm{CH}_{2}$ $\mathrm{Cl}_{2}$. Next, the $\mathrm{CH}_{2} \mathrm{Cl}_{2}$ fraction was concentrated and reprecipitated with $\mathrm{CH}_{3} \mathrm{OH}$. The dark-green solid was gathered and dried under a vacuum at $45^{\circ} \mathrm{C}$, which was confirmed by the ${ }^{1} \mathrm{H}-\mathrm{NMR}$ (Figure $\mathrm{S} 1$ ).

\section{Preparation of PD-FA Nanoparticles}

PIIGDTS photothermal material $2 \mathrm{mg}$, PCL-PEG-FA $5 \mathrm{mg}$, and Ps-S-PEG $15 \mathrm{mg}$ were completely dissolved in $2 \mathrm{~mL}$ of tetrahydrofuran. Then, the mixture was added drop by drop to $10 \mathrm{~mL} \mathrm{ddH}_{2} \mathrm{O}$ under the action of 
a magnetic stirrer and stirred in the ventilator for more than 12 hours. Solution was collected and filtered by filter paper, and the nanoparticles were obtained by ultrafiltration with an ultrafiltration tube.

\section{Photothermal Ability of the PD-FA Nanoparticles}

To assess the photothermal ability of the PD-FA nanoparticles, the temperature of nanoparticles with different concentrations ranging from 0 to $80 \mu \mathrm{g} \mathrm{mL}^{-1}$ was monitored using the infrared thermal imaging camera (CEM, Beijing) at an interval of 60 seconds. A PD-FA nanoparticle aqueous solution $(0.5 \mathrm{~mL})$ was introduced into Eppendorf Micro Test Tubes. Later, the tubes were irradiated with an $845 \mathrm{~nm}$ laser (Changchun New Industries Optoelectronics Technology Co., Ltd., China) for $10 \mathrm{~min}-$ utes. The PD-FA nanoparticles $\left(80 \mu \mathrm{g} \mathrm{mL}^{-1}\right)$ were exposed to different power intensities with an $845 \mathrm{~nm}$ laser, including $0.5 \mathrm{~W} \mathrm{~cm}^{-2}, 1 \mathrm{~W} \mathrm{~cm}^{-2}$, and $1.5 \mathrm{~W} \mathrm{~cm}^{-2}$. Pure water irradiated with the same laser under $1.5 \mathrm{~W} \mathrm{~cm}^{-2}$ was used as a negative control. The temperature of the solution was recorded to evaluate the photothermal conversion efficiency. Finally, to investigate the photostability of PD-FA nanoparticles, $0.5 \mathrm{~mL}$ of PD-FA nanoparticles underwent five cycles of photothermal heating and natural cooling under $845 \mathrm{~nm}$ laser irradiation at the intensity of 1.5 $\mathrm{W} \mathrm{cm}{ }^{-2}$.

\section{PAI System}

The photoacoustic properties of the PD-FA nanoparticles were evaluated by our MSPAM system. ${ }^{53}$ In our MSPAM system (Figure S2A), the energy source used to excite the materials was a wavelength tunable nanosecond pulsed OPO laser (Continuum, USA), and an ultrasound transducer with a central frequency of $25 \mathrm{MHz}$ (Olympus, USA) was used to detect the generated acoustic signal. Then, the obtained signals were amplified by an amplifier (Olympus, USA). The data acquisition module and motorized 2D scanning stage were also used to construct the system. We tested and verified the imaging resolution and imaging depth of our MSPAM system by series of experiments. To describe the resolution of our system, three pieces of human hair with a diameter of $\sim 100 \mu \mathrm{m}$ were imaged. The hair was embedded and stabilized in agar. The opticalabsorption map of the agar-hair phantom is displayed in a maximum intensity projection (MIP) image with high contrast in Figure S2B. An image profile along the red dashed line from the MIP image is provided in Figure S2C. These results demonstrated that the system could resolve micrometer-scale structures with high contrast. Besides, agar (2\%) and water were heated and added to the lipid (1\%) to form emulsion for solid model, a copper wire immersed in ink was inserted in the solid model and was imaged by our MSPAM system. As shown in Figure S3, the deepest depth of the copper wire which could be imaged is up to $2.2 \mathrm{~mm}$. This result validated the imaging depth of our MSPAM system.

\section{Photoacoustic Performance of PD-FA Nanoparticles}

The photoacoustic spectrum of PD-FA nanoparticles was obtained by recording the photoacoustic signals of 80 $\mu \mathrm{g} \mathrm{mL} \mathrm{L}^{-1}$ PD-FA nanoparticles injected into a transparent glass tube fixed in agar with the interval of $10 \mathrm{~nm}$ ranging from $760 \mathrm{~nm}$ to $960 \mathrm{~nm}$. The photoacoustic intensities were measured 50 times at each wavelength. Then, the photoacoustic stability of the PD-FA nanoparticles was estimated by monitoring the variation in photoacoustic intensity under the continuous irradiation of an $845 \mathrm{~nm}$ laser at the same energy density for 20 minutes. The photoacoustic images and intensities of PD-FA nanoparticles with different concentrations $(0,10,20,40$ and 80 $\mu \mathrm{g} \mathrm{mL} L^{-1}$ ) were obtained as mentioned above under the same conditions.

\section{Cell Culture}

Cell lines for in vitro cell experiment were purchased from the American Type Culture Collection (ATCC). The environment contained $5 \% \mathrm{CO}_{2}$ and $95 \%$ air at $37^{\circ} \mathrm{C}$. HeLa cells and cos-7 cells were cultured in Dulbecco's Modified Eagle Medium (DMEM, Gibco) with 10\% fetal bovine serum (FBS, BI) and $1 \%$ penicillin/streptomycin (Corning).

\section{Cell Uptake of the PD-FA Nanoparticles}

To detect the process of cellular uptake of the PD-FA nanoparticles, Coumarin-6 (CU-6) with near-infrared fluorescence emission was encapsulated in the PD nanoparticles and PD-FA nanoparticles. The HeLa cells were cultured in confocal dishes and adhered for 24 hours, then cocultured with PD nanoparticles or PD-FA nanoparticles containing CU-6 for 2, 4, and 8 hours. At each time point, the cells were washed with PBS three times and fixed with a $4 \%$ paraformaldehyde solution for 10 minutes. Finally, the cells were stained by 4',6-diamidino-2-phenylindole 
(DAPI) for approximately 10 minutes. Fluorescence images were further obtained by a confocal laser scanning microscope (Leica TCS SP8, Germany). All the procedures were carried out in the dark.

\section{In vitro Cytotoxicity Test of PD-FA Nanoparticles Before and After Excitation}

To investigate the cytotoxicity of the as-obtained PD-FA nanoparticles for normal cells, MTT assay was used for in vitro cell activity evaluation. Cos- 7 cells $\left(7 \times 10^{3}\right.$ cells/ per well, $100 \mu \mathrm{L}$ ) were seeded on 96-well plates for 12 hours. Then, the media were removed, and the cells in wells were incubated with a PD-FA nanoparticles aqueous solution at various concentrations $(0,5,10,20,40,80$ $\mu \mathrm{g} \mathrm{mL}{ }^{-1}$ ) for 24 hours. After 24 hours of incubation at $37^{\circ} \mathrm{C}$, MTT $\left(20 \mu \mathrm{L}, 5000 \mu \mathrm{g} \mathrm{mL}^{-1}\right)$ was added, and the cells were incubated in each well for 4 hours. The absorbance (OD: $570 \mathrm{~nm}$ ) of each well was measured by an enzyme labeling apparatus, and the cell viability was obtained.

To investigate the cytotoxicity of the as-obtained PDFA nanoparticles for Hela cells after excitation, PD and PD-FA nanoparticles were diluted to various concentrations using DMEM. Then, the Hela cells were incubated in 96-well plates and divided into three groups: (1) PD nanoparticles + Laser; (2) PD-FA nanoparticles + HeLa pretreated with free aptamer + Laser;, (3) PD-FA nanoparticles + Laser. After 8 hours of incubation, the cell culture media were removed. MTT $(20 \mu \mathrm{L}, 5000$ $\mu \mathrm{g} \mathrm{mL}^{-1}$ ) in new cell culture media were added, and the cells were incubated in each well for 4 hours. The absorbance (OD: $570 \mathrm{~nm}$ ) of each well was measured by an enzyme labeling apparatus, and the cell viability was obtained.

\section{Calcein-AM/PI Cell Staining}

For cell viability detection after exposure to the laser, the cells were co-cultured with a live/dead cell double staining kit to monitor viable and dead cells with the confocal fluorescence microscope. The double staining kit contains Calcein-AM, which stained only viable cells with green fluorescence, and propidium iodide (PI), which stained only dead cells with red fluorescence. After being seeded in 96-well plates $\left(5 \times 10^{4}\right.$ cells per well) and adhering for 24 hours, the cells were divided into six groups with different treatment methods: (1) PD-FA nanoparticles + Laser; (2) PD-FA nanoparticles + HeLa pretreated with free aptamer + Laser; (3) PD nanoparticles + Laser; (4) PD-FA nanoparticles only; (5) Laser only; (6) Control. After 8 hours of incubation, the media were abandoned and the cells were washed three times with PBS. After laser $(845 \mathrm{~nm})$ irradiation, the cells were cultured at $37^{\circ} \mathrm{C}$ with $5 \% \mathrm{CO}_{2}$ and $95 \%$ air for 24 hours. Then, the fresh DMEM containing the live/dead cell double staining kit was added to all wells and further cultured for 30 minutes. Finally, the fluorescent images of all groups were acquired by a fluorescence microscope (Leica, Germany).

\section{Animals and Tumor Model}

All animal procedures were performed in accordance with the Guidelines for Institutional Animal Care and Use Committee and approved by the approval of the Animal Care and Usage Committee of Guangzhou Medical University (GY2019-138). BALB/c nude mice used in all experiments were purchased from SPF (Beijing) Biotechnology Co., Ltd. Five-week-old $\mathrm{BALB} / \mathrm{c}$ nude mice were selected to establish the tumor model for in vivo PAI and PTT. The tumor cells were cultivated according to the procedure mentioned above. The subcutaneous tumor models were developed by injecting $5 \times 10^{6}$ cells in $0.1 \mathrm{~mL}$ PBS subcutaneously on the back of the mice. The in vivo experiments were conducted when the tumor size reached $100 \mathrm{~mm}^{3}$ (tumor volume was calculated by the formula $\mathrm{V}=a b^{2} / 2$, where $a$ and $b$ are the tumor's length and width, respectively).

\section{In vivo PAI}

To evaluate the in vivo photoacoustic performance of the PD-FA nanoparticles or PD nanoparticles for the HeLa subcutaneous tumor, the HeLa tumor-bearing nude mice were intravenously injected with the as-prepared nanoparticles $\left(200 \mu \mathrm{L}, 2000 \mu \mathrm{g} \mathrm{mL}^{-1}\right)$. For PAI, tumor-bearing mice with intravenously injected nanoparticle aqueous solution were anesthetized (with $1 \%$ sodium pentobarbital). The photoacoustic images of the tumor site were obtained at different time intervals $(2,4,8,12,24$ and 36 hours) and were captured by our MSPAM system with $845 \mathrm{~nm}$ laser excitation. 


\section{In vivo PTT and Histological Analysis}

To evaluate the photothermal properties of PD-FA nanoparticles in HeLa tumor-bearing nude mice, thirty mice in which the volume of the tumor reached $100 \mathrm{~mm}^{3}$ were selected for in vivo PTT. These mice were randomly divided into five groups ( $n=6$ per group) as follows: (1) PD-FA nanoparticles + Laser; (2) PD nanoparticles + Laser; (3) PD-FA nanoparticles only; (4) Laser only; (5) Control. All mice were treated with the laser under the same parameters $\left(845 \mathrm{~nm}, 1 \mathrm{~W} \mathrm{~cm}^{-2}, 5\right.$ minutes). The tumor volumes and body weights of all tumorbearing nude mice were monitored every three days. After therapy, the mice from each group were finally sacrificed to collect the tumors and five major organs including the heart, liver, spleen, lung and kidneys for H\&E staining.

\section{A}

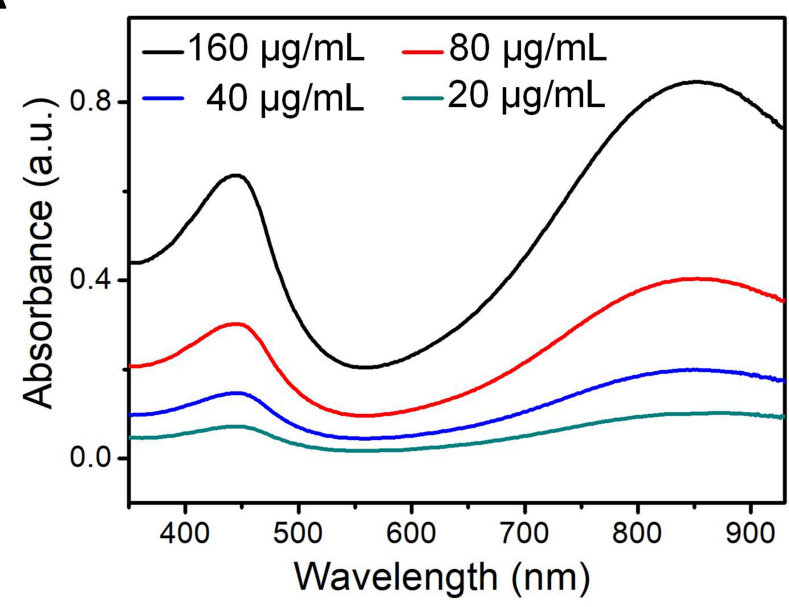

C

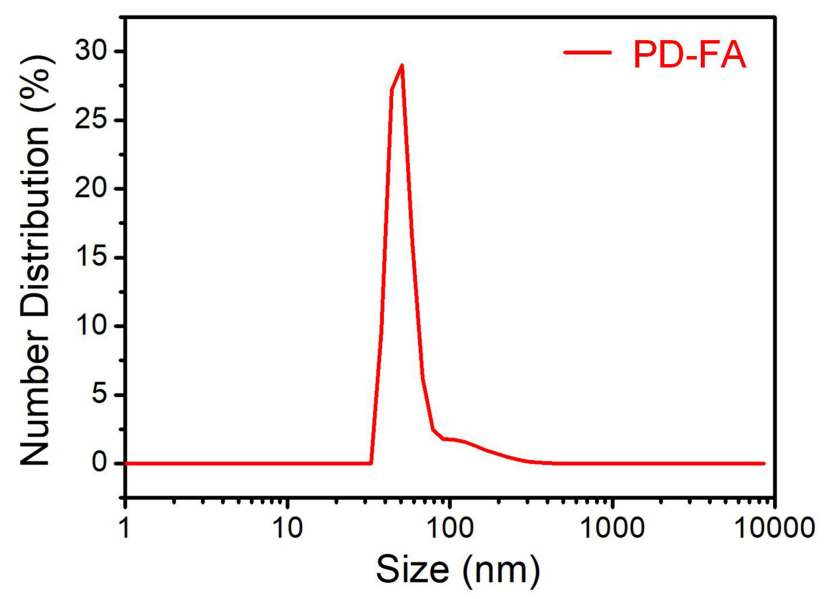

\section{Results and Discussion}

Synthesis and Characterization of the PD-FA Nanoparticles

We designed PD-FA nanoparticles that have active targeting ability for cervical cancer by one-step nanoprecipitation method to simultaneously facilitate photoacoustic enhancement and the effect of PTT.

The absorption spectra of the PD-FA nanoparticles were recorded as shown in Figure 2A. The PD-FA nanoparticles displayed a broad absorption spectrum from the visible to NIR region. This indicated that for two characteristic peaks, at $450 \mathrm{~nm}$ and $845 \mathrm{~nm}$, the former is mainly due to $\pi-\pi^{*}$ transition of the polymer backbone, ${ }^{54}$ while the latter is ascribed to the optical absorption of PD-FA

B

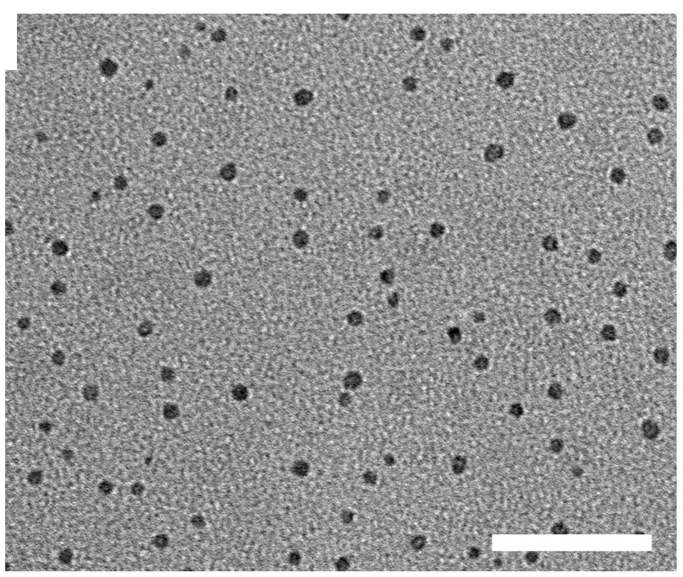

D

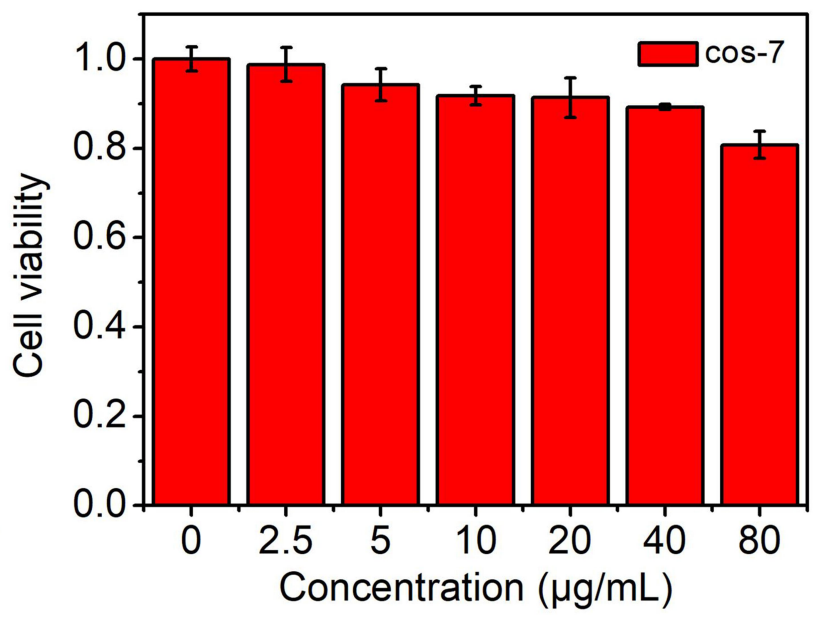

Figure 2 Characterization and cytotoxicity of the as-prepared PD-FA nanoparticles. (A) UV-Vis absorption spectra of the PD-FA nanoparticles at different concentration dissolved in water. (B) Transmission Electron Micrograph (TEM) image of PD-FA nanoparticles with an average size of $9 \pm 3 \mathrm{~nm}$. The scale bar is $50 \mathrm{~nm}$. (C) Hydrodynamic diameter of the as-prepared PD-FA nanoparticles dispersed in PBS measured by dynamic light scattering (DLS). (D) Cell viabilities of cos-7 cells incubated with the asprepared PD-FA nanoparticles at various concentrations ranging from $0 \mu \mathrm{g} \mathrm{mL} \mathrm{L}^{-1}$ to $80 \mu \mathrm{g} \mathrm{L}^{-1}$. 
nanoparticles in NIR. With the broadband absorption spectrum, the PD-FA nanoparticles can satisfy different demands according to various requirements. The morphology and size of the PD-FA nanoparticles were then measured by TEM. As observed in Figure 2B, the TEM images of PD-FA nanoparticles showed a regular spherical structure with an average diameter of approximately $9 \mathrm{~nm}$ evenly distributed in the solution, which exhibited good monodispersity of the PD-FA nanoparticles. In addition, the hydrodynamic diameter of the PD-FA nanoparticles was determined using DLS. As shown in Figure $2 \mathrm{C}$, the hydrophilic diameter reached $50 \mathrm{~nm}$, which was bigger than that measured in the TEM image. This difference is mainly attributed to the different measuring condition since the TEM represented the diameter of PD-FA nanoparticles that was dried on the copper grid while the DLS measured the diameter in the aqueous solution. ${ }^{55}$ For the following potential in vivo biological applications, the biocompatibility at the cellular level of the as-prepared PD-FA nanoparticles was also determined. The cytotoxicity was evaluated by testing the cell viability of the cos- 7 cell when co-cultured with different concentrations of the PD-FA nanoparticles ranging from 0 to $80 \mu \mathrm{g} \mathrm{mL}^{-1}$ at 24 hours. After 24 hours, incubation of HeLa cells with PDFA nanoparticles, no significant cytotoxicity was noticed even at $80 \mu \mathrm{g} \mathrm{mL}^{-1}$, demonstrating good biocompatibility of the PD-FA nanoparticles (Figure 2D). These results revealed that the PD-FA nanoparticles have strong NIR absorption, suitable particle sizes, good biostability and excellent biocompatibility.

\section{Photothermal Property and Stability of the PD-FA Nanoparticles}

The photothermal potential of the PD-FA nanoparticles was further surveyed by monitoring the temperature variation under laser irradiation. The thermographic images of PD-FA nanoparticles solutions under the continuous irradiation of $845 \mathrm{~nm}$ laser for 10 minutes obtained by an infrared thermal imaging camera are exhibited in Figure $3 \mathrm{~A}$. It is obvious that the temperature was dramatically raised with the irradiation time, which demonstrated that the PD-FA nanoparticles were able to efficiently convert absorbed photon energy into heat energy that kills HeLa cells. To further investigate the photothermal property of PD-FA nanoparticles, we quantitatively monitored the exact temperature changes in PD-FA nanoparticles with different concentrations $\left(0,5,10,20,40\right.$ and $\left.80 \mu \mathrm{g} \mathrm{mL}^{-1}\right)$ under the irradiation of $1.5 \mathrm{~W} \mathrm{~cm}^{-2}$ and different laser powers $\left(0.5,1,1.5 \mathrm{~W} \mathrm{~cm}^{-2}\right)$ at the concentration of 80 $\mu \mathrm{g} \mathrm{mL}^{-1}$. As displayed in Figure 3B, under the laser irradiation, the temperature elevation rate was considerably fast in the first 4 minutes and became slower until it reached zero in the 7 th minute. Finally, the temperature variation reached $80^{\circ} \mathrm{C}$ at the concentration of $80 \mu \mathrm{g} \mathrm{mL}^{-1}$ under the irradiation of an $845 \mathrm{~nm}$ laser $\left(1.5 \mathrm{~W} \mathrm{~cm}^{-2}\right)$, while the water used as a control displayed negligible temperature increments under the same laser irradiation conditions. It was found that the ultimate temperature variation was dependent on the concentration of the PDFA nanoparticles. The laser power dependence was shown in Figure 3C, and it was not difficult for us to observe that the temperature of PD-FA nanoparticles solution could increase to $43^{\circ} \mathrm{C}$, even at a relatively low laser power density for effective tumor ablation.

The stability of nanoparticles is of great significance for the application in biological fields. The photothermal stability of PD-FA nanoparticles was verified by monitoring the thermal curve of five heating and cooling cycles under NIR laser irradiation $\left(1.5 \mathrm{~W} \mathrm{~cm}^{-2}\right)$. Unexpectedly, the PD-FA nanoparticles still promoted the temperature at the same level, even after five irradiation cycles, and the trend of the curve remained unchanged in each cycle (in Figure 3D), with the resulting conclusion that the asprepared PD-FA nanoparticles possess excellent photostability. The same experiment has also been taken in the US Food and Drug Administration (FDA)-approved indocyanine green (ICG) dye that is widely applied in the biomedical field. It is noted that the temperature was gradually decreased after one irradiation cycle in Figure $\underline{\mathrm{S}}$. This phenomenon may be ascribed to the weakening of the NIR absorbing ability of ICG. We verified this assumption, which showed that the absorption of ICG was greatly diminished after laser irradiation (Figure S5). The insets also show obvious fading from light green to yellow in the ICG solution. Additionally, the storage stability of PD-FA nanoparticle was measured by taking photographs of the PD-FA nanoparticle dispersed in water and storage in the ambient environment for five and ten days. As shown in Figure S6A, no aggregation could be found after storage in the ambient environment for ten days and it remained a transparent deep green. Besides, the spectral signal exhibited a negligible decrease in Figure S7. The results indicated the stability of PD-FA nanoparticles in long-term storage. As the PD-FA nanoparticles were injected to blood and accumulated in tumor, the stability of PD-FA 
A

0
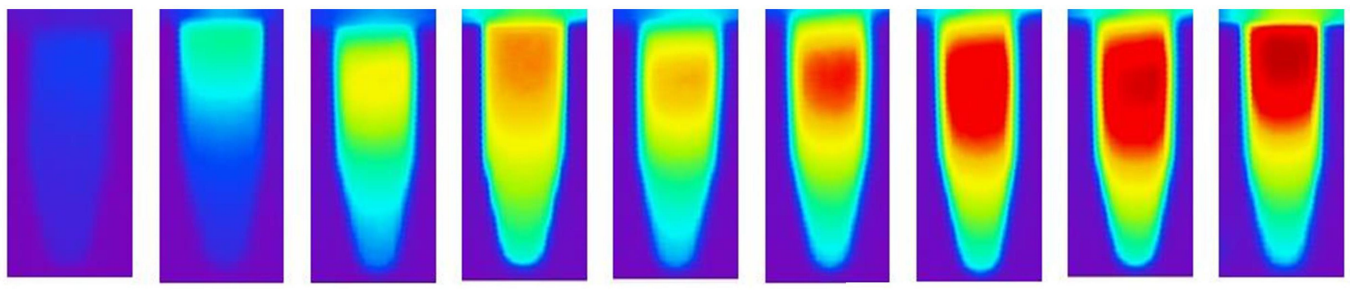

B

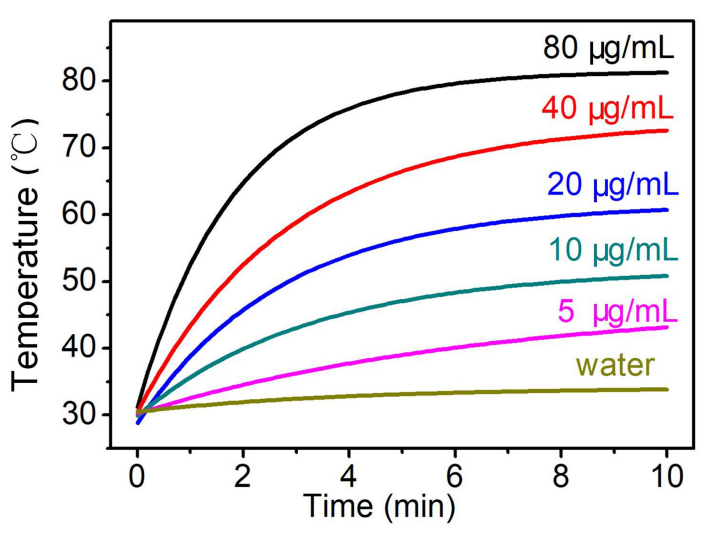

D

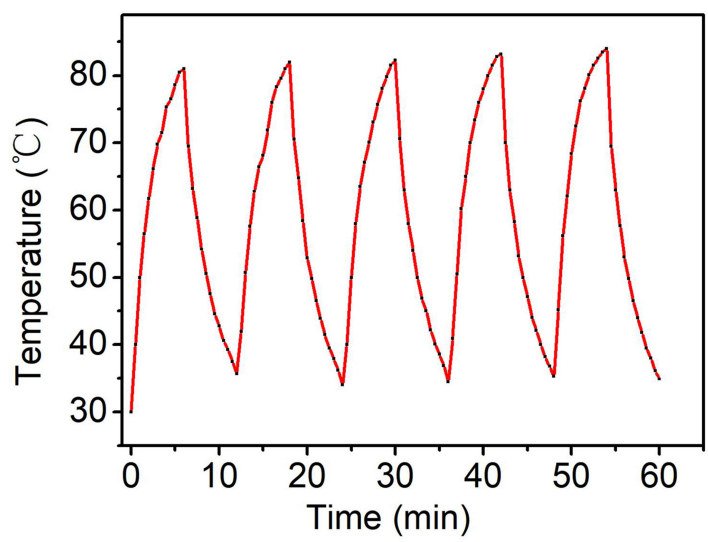

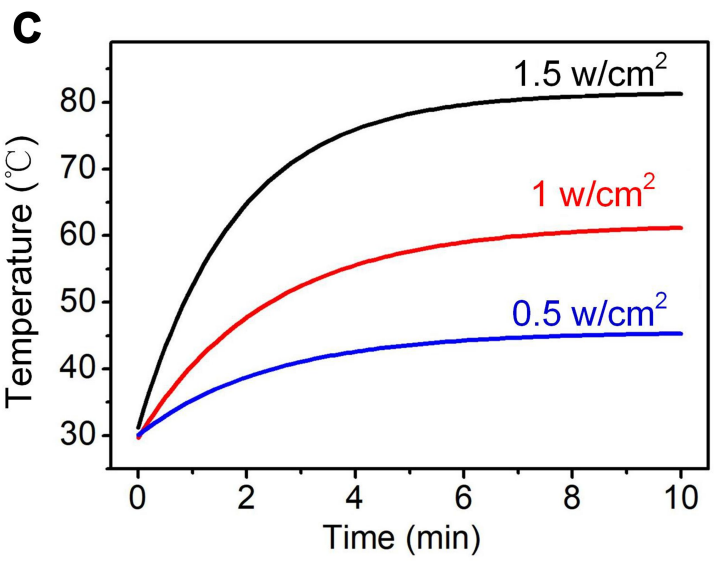

$\mathbf{E}$

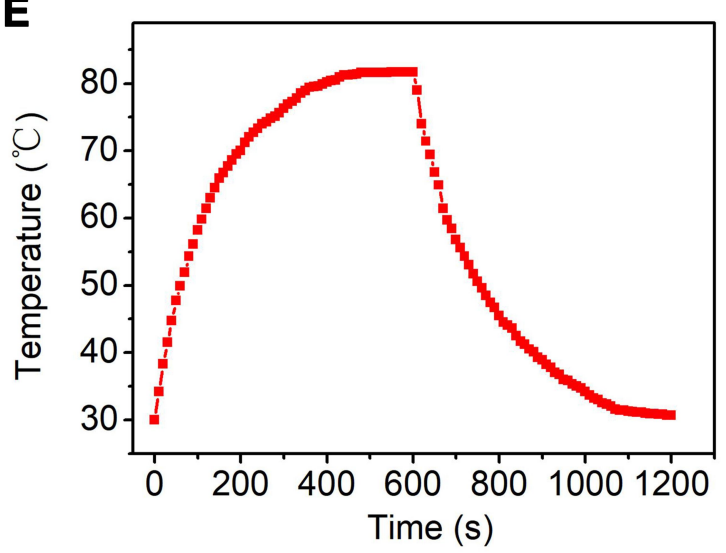

Figure 3 Photothermal properties of PD-FA nanoparticles in vitro. (A) A series of photothermal images of PD-FA nanoparticles solution $\left(80 \mu g \mathrm{~mL}^{-1}\right)$ under the irradiation of $845 \mathrm{~nm}$ laser at the power density of $1.5 \mathrm{~W} \mathrm{~cm}^{-2}$. (B) The temperature variation of the solution containing different concentrations of the PD-FA nanoparticles (0, 5,10 , $20,40,80 \mu \mathrm{g} \mathrm{mL}^{-1}$ ) under the irradiation of $845 \mathrm{~nm}$ laser at the power density of $1.5 \mathrm{~W} \mathrm{~cm}{ }^{-2}$. (C) The temperature curves of PD-FA nanoparticles at the concentration of $80 \mu \mathrm{gL}^{-1}$ under $845 \mathrm{~nm}$ laser irradiation at different power densities. (D) Temperature variations of the PD-FA nanoparticles under $845 \mathrm{~nm}$ laser irradiation at a power density of $1.5 \mathrm{~W} \mathrm{~cm}^{-2}$ for five light on/off cycles laser irradiation. (E) Photothermal effect of PD-FA nanoparticles in water when irradiated with a $845 \mathrm{~nm}$ laser (I.5 $\mathrm{W} \mathrm{cm}$ ). The laser was switched off after irradiation for $10 \mathrm{~min}$.

nanoparticles in blood and acidic environment of tumor was further measured. There was no aggregation can be found when PD-FA nanoparticles were dispersed in PBS $(\mathrm{pH}=4.5)$ and FBS in Figure S6B, which resulted that PD-FA nanoparticles stayed stable in blood and acidic environment.

To quantitatively access the photothermal conversion capability, we explored the photothermal conversion efficiency ( $\eta)$ of PD-FA nanoparticles. The PD-FA nanoparticles suspension $\left(80 \mu \mathrm{g} \mathrm{mL}^{-1}, 0.5 \mathrm{~mL}\right)$ was irradiated with the NIR laser $\left(845 \mathrm{~nm}, 1.5 \mathrm{~W} \mathrm{~cm}^{-2}\right)$ until the temperature reached a steady state, and then the laser was shut off and the system was cooled naturally. The photothermal conversion efficiency was deduced from the heating and cooling temperature curves (Figure 3E). According to the standard method proposed previously in the literature, ${ }^{36}$ the photothermal conversion efficiency of the PD-FA nanoparticles was calculated to about $62.6 \%$. 
Taking above results into overall consideration, the performance of PD-FA nanoparticles with good stability and admirable photothermal conversion efficiency were concentration-, power- and time-dependent to satisfy the demand of patients with cervical cancer at different stages, which suits the concept of precision medicine.

\section{In vitro Photoacoustic Ability of the PD-FA Nanoparticles}

To confirm the photoacoustic properties of PD-FA nanoparticles, related experiments on as-prepared PD-FA nanoparticles were conducted by our MSPAM system introduced previously. First, we measured the photoacoustic signal of PD-FA nanoparticles at wavelengths ranging from $760 \mathrm{~nm}$ to $960 \mathrm{~nm}$, suggesting that the PD-FA nanoparticles exhibited a strong photoacoustic signal covering the NIR region of $850 \mathrm{~nm}$ to $900 \mathrm{~nm}$ with a characteristic peak at $875 \mathrm{~nm}$. It is obvious that the tendency of the photoacoustic spectrum (shown in Figure 4A) almost corresponded with the absorption spectrum. However, the peak in the photoacoustic spectrum was blue-shifted compared with the absorption spectrum, a phenomenon that has been observed in other organic photoacoustic agent ${ }^{56}$ and that is mainly ascribed to the effect of the chemical structure of the nanoparticles or to the differences in the optical illumination parameters and photophysical processes of the two spectra. Next, the photo-stability of the PD-FA nanoparticles was also evaluated by recording the change in photoacoustic signal under laser irradiation. As displayed in Figure 4B, there was no obvious change in photoacoustic signals during the 20 minutes laser irradiation, which indicated the stability of the enhancement of photoacoustic imaging utilizing the PD-FA nanoparticles. After confirming the best wavelength for imaging and the photostability of PD-FA nanoparticles, we performed PAI of PD-FA nanoparticles at different concentrations (10, 20, 40 and $80 \mu \mathrm{g} \mathrm{mL}^{-1}$ ) under laser ( $845 \mathrm{~nm}$ ) excitation. Then, the last photoacoustic photograph was obtained by our MSPAM system. It can be observed in Figure 4C that the PD-FA nanoparticles exhibited a photoacoustic effect even at a low concentration. As shown in Figure 4D, the solution was added to a glass tube with a diameter of $0.9 \mathrm{~mm}$ that was embedded in an agar block. Moreover, the photoacoustic signals dramatically increased with the increase in PD-FA nanoparticle concentrations. The imaging profiles along with the green dashed line from the photoacoustic image are displayed in Figure 4E, the five sharp peaks represent the photoacoustic signal of different concentrations of the PD-FA nanoparticles. A good linear relationship ( $r=0.968$ ) between the photoacoustic signals and the concentrations of PD-FA nanoparticles can also be found in Figure 4F. Therefore, the above results show that the PD-FA nanoparticles exhibited desirable photoacoustic performance in vitro.

\section{Photothermal Effect of the Nanoparticles at the Cellular Level}

Motivated by the exciting photothermal efficacy of the asprepared PD-FA nanoparticles, its photothermal cell damage effect was estimated on HeLa cells to prepare for the further living animal experiment. First, the cellular uptake of the PD-FA nanoparticles was validated. Coumarin-6 (CU-6), a hydrophobic molecule with fluorescence emission, was encapsulated in the nanoparticles (namely, PD-CU-6 nanoparticles and PD-FA-CU-6 nanoparticles) so that the nanoparticles could be monitored by fluorescence microscopy. The HeLa cell nuclei were dyed with DAPI (blue) and the nanoparticles with CU-6, which presents green fluorescence signals. As displayed in Figure $5 \mathrm{~A}$, green fluorescence signals appeared after incubation with the PD-FA-CU-6 nanoparticles for 2 hours in the HeLa cell and gradually became stronger with time and achieved a high level in 8 hours, while there was almost no fluorescence signal when the cells were incubated with nanoparticles without FA (PD-CU-6 nanoparticles) at 2 hours, 4 hours, and even at 8 hours, suggesting that PDFA nanoparticles have good selectivity toward HeLa cells.

Based on the excellent targeting ability of PD-FA nanoparticles to HeLa cells, the targeted photothermal killing capability of the PD-FA nanoparticles was investigated in the next step. HeLa cells were differently treated and irradiated under NIR laser at $1 \mathrm{~W} \mathrm{~cm}^{-2}$ for 10 minutes. Six groups were prepared as followed: (a) PD-FA nanoparticles + Laser; (b) PD-FA nanoparticles + HeLa pretreated by free FA+ Laser; (c) PD nanoparticles + Laser; (d) PD-FA nanoparticles; (e) Laser only; (f) Control. Free FA was mixed with cell culture medium and incubated with HeLa cells one hour in advance as a competitor with PD-FA nanoparticles. To visualize the cell survival state after different treatments, we employed the live/dead cell double-staining kit in which Calcein-AM stains live cells with green fluorescence and PI stains dead cells with red fluorescence so that we could directly observe the cell condition through a confocal fluorescence microscope. As 
A

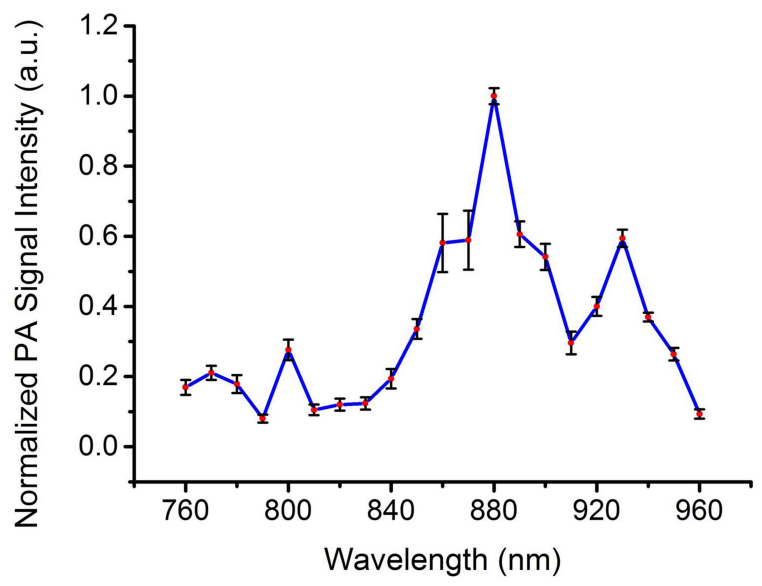

C

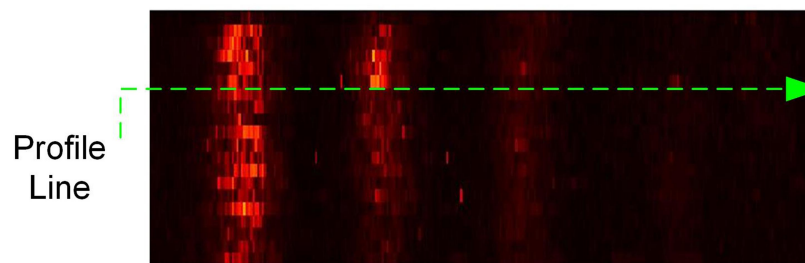

$80 \mu \mathrm{g} / \mathrm{mL} \quad 40 \mu \mathrm{g} / \mathrm{mL} 20 \mu \mathrm{g} / \mathrm{mL} 10 \mu \mathrm{g} / \mathrm{mL}$ Water
B

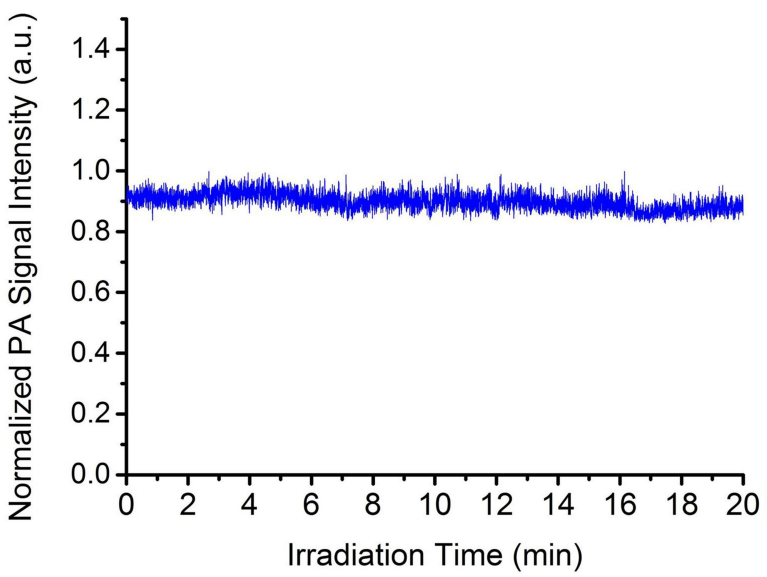

$\operatorname{Max}$

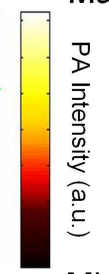

Min
D

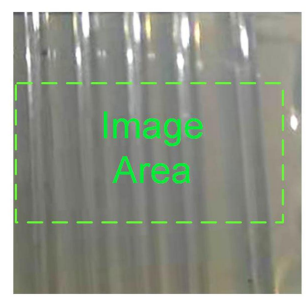

E

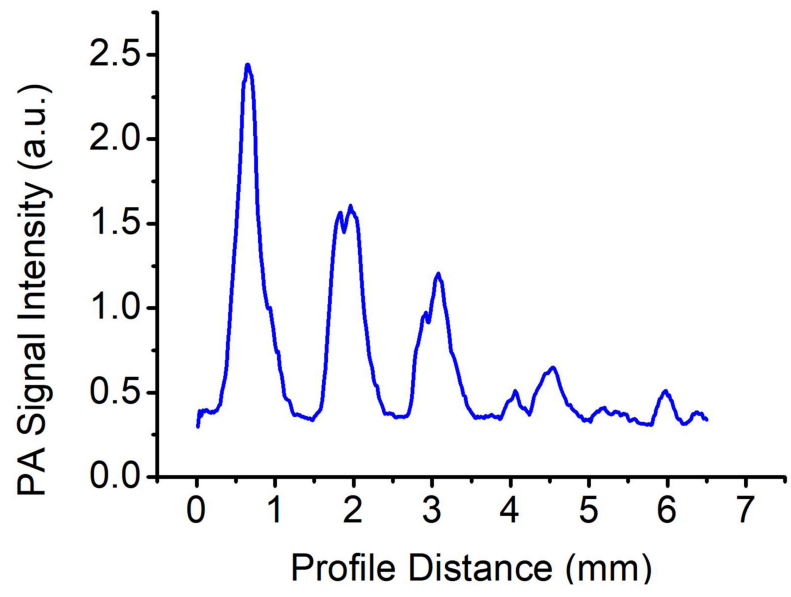

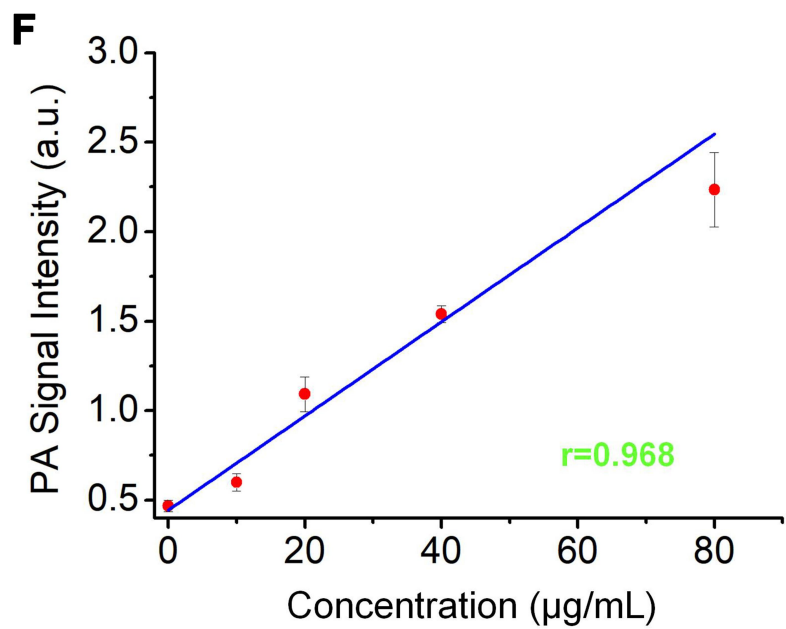

Figure 4 In vitro photoacoustic property of as-prepared PD-FA nanoparticles. (A) Photoacoustic spectrum of PD-FA nanoparticles at different wavelengths. (B) The photostability of photoacoustic signal under the irradiation of different laser powers for 20 min. (C) Photoacoustic images of PD-FA nanoparticles nuder excitation at 845 $\mathrm{nm}$ at different concentrations. (D) Photograph of sample in agar phantom. (E) Intensity profiles along the dashed line from (C). (F) The Photoacoustic amplitudes at $845 \mathrm{~nm}$ as a function of concentrations of PD-FA nanoparticles.

displayed in Figure 5B, HeLa cells in group (a) all displayed a strong red fluorescence signal and no green fluorescence signal, indicating the HeLa cell-killing abilities of the PD-FA nanoparticles. However, the cell damage effect was distinctly reduced in group (b) and group (c), further indicating the specific HeLa tumortargeting properties of the PD-FA nanoparticles. As a negative control, the HeLa cells under a single factor (only laser or only PD-FA nanoparticles) and no treatment showed almost no red fluorescence, thereby implying no therapeutic effect for the HeLa cells. To accurately estimate the cell damage efficacy of the PD-FA nanoparticles, the cell viabilities of the HeLa cervical cancer cells were measured after treatment with PD-FA nanoparticles plus laser, PD nanoparticles plus laser and pretreated with free FA before PD-FA nanoparticles plus laser. As presented in 


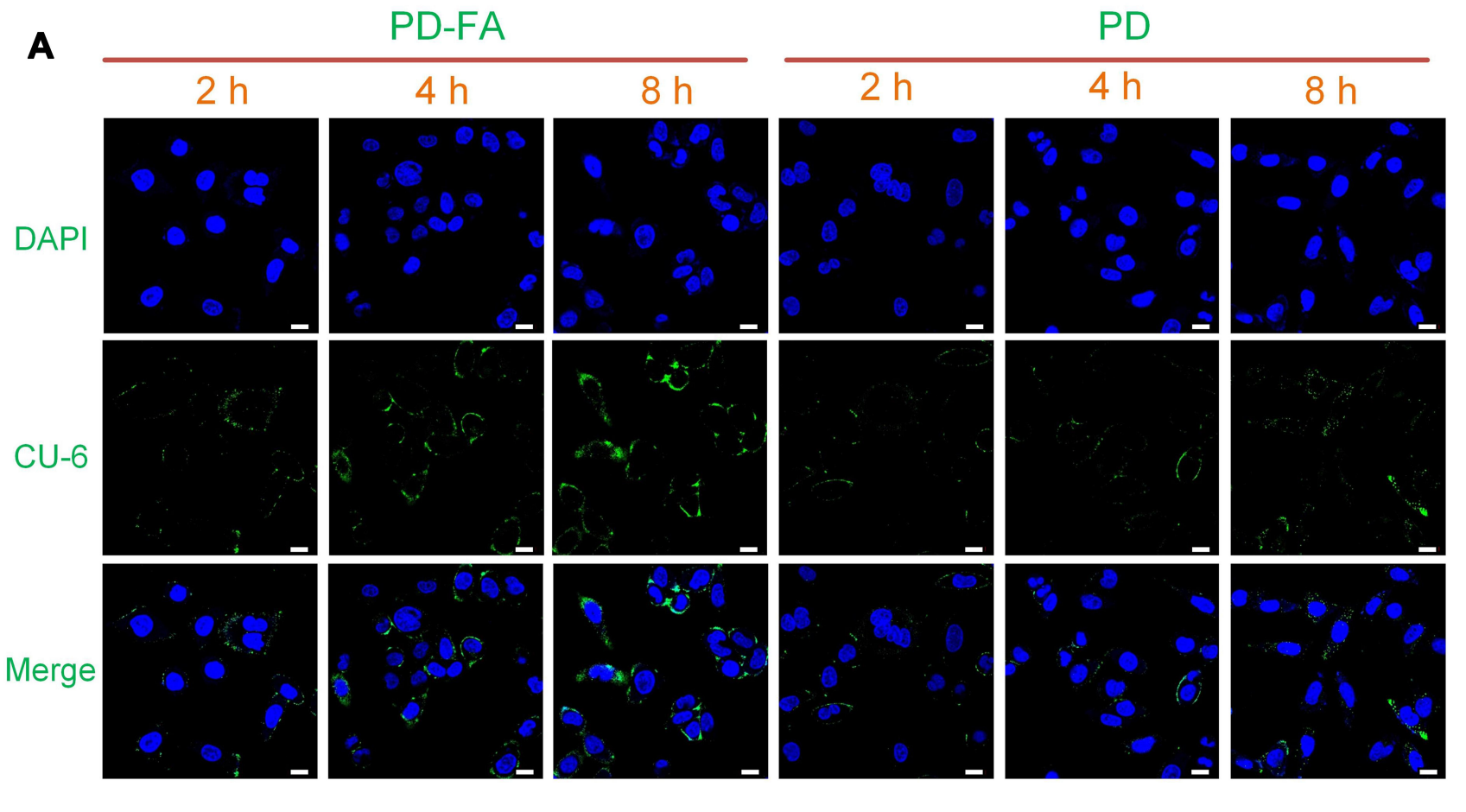

B
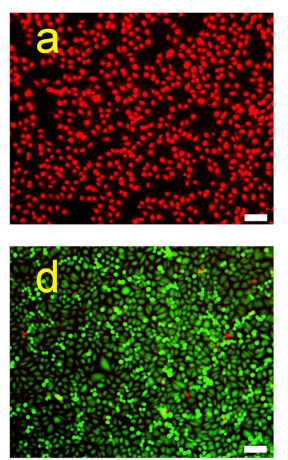
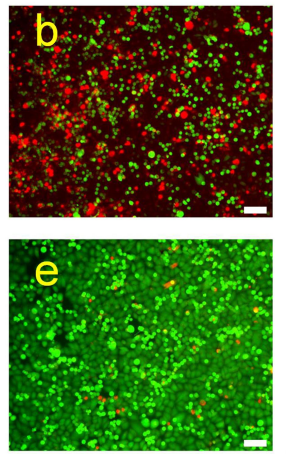
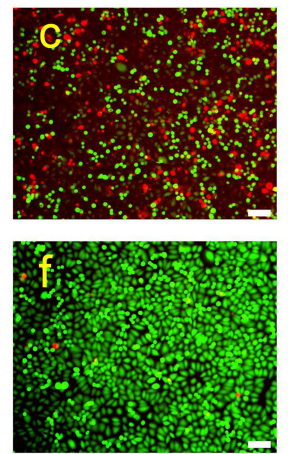

C

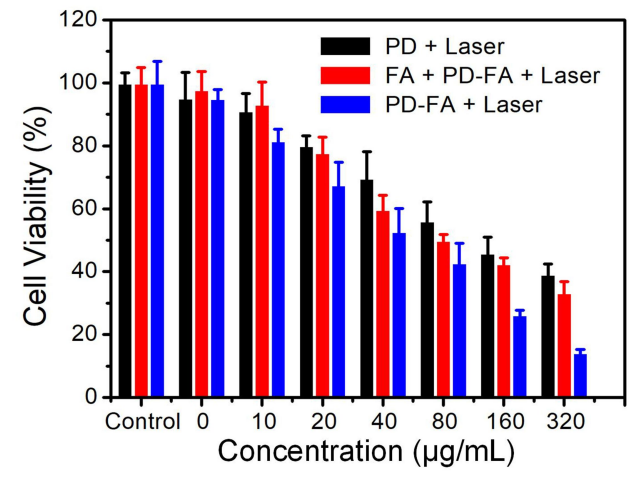

Figure 5 Cell uptaking and photothermal abilities incubated with HeLa cells of PD-FA nanoparticles and PD nanoparticles. (A) Confocal fluorescence images of HeLa cancer cells co-cultured with PD-FA nanoparticles and PD nanoparticles for 2, 4, $8 \mathrm{~h}$. Scale bar is $20 \mu \mathrm{m}$. (B) Fluorescence images of Calcein AM (green, live cells) and propidium iodide (red, dead cells) contained HeLa cells after different treatments including; (a) PD-FA nanoparticles + Laser; (b) PD-FA nanoparticles + HeLa pre-treated by free FA+ Laser; (c) PD nanoparticles + Laser; (d) PD-FA nanoparticles, (e) Laser only; (f) Control (845 nm laser irradiation at a power density of I W $\mathrm{cm}^{-2}$ for 10 min). Scale bar is 50 $\mu \mathrm{m}$. (C) The cell viabilities of the HeLa cervical cancer cells after treatment with PD-FA nanoparticles plus laser, PD nanoparticles plus Laser and pre-treated by free FA before PD-FA nanoparticles plus laser.

Figure 5C, the cell mortality of the HeLa cells was over $50 \%$ when incubated with a relatively low concentration of the PD-FA nanoparticles at the laser irradiation and increased with incremental concentration increases. It was notable that the cell mortality reached $80 \%$ when HeLa cells were treated with $320 \mu \mathrm{g} \mathrm{mL}^{-1}$ PD-FA nanoparticles. Moreover, the cell mortality was reduced when the cells were incubated with PD nanoparticles plus laser or PD-FA nanoparticles plus laser pretreated with free FA. The receptors of FA are highly expressed on the surface of Hela cells, free HA was used as a competitor with PD-FA nanoparticles on the intracellular cell uptake experiment, which reduced the cellular uptake of PD-FA nanoparticles into the cells. Then, FA + PD-FA + laser group has relatively weak cell-killing effect than PD-FA + laser group. That is, FA + PD-FA + laser has higher cell viability than PD-FA + laser. This result further proved the targeted cell killing ability of PD-FA after excitation.

To intuitively observe the influence of concentration and laser power intensity on the PD-FA nanoparticles, HeLa cells with different concentrations of PD-FA nanoparticles under various laser power intensities were co- 
stained by a live/dead cell double-staining kit and viewed with a fluorescence microscope. It could be observed in Figure S8 that the PD-FA nanoparticles exhibited a remarkable cell killing capacity, even at low power intensity. These results further demonstrated the concentration-dependence and power-dependence of the PD-FA nanoparticles. Therefore, we verified the active targeting of HeLa cells by the PD-FA nanoparticles and evaluated the admirable efficacy of PTT in vitro.

\section{In vitro and in vivo PAI of Nanoparticles}

According to the above results, the PAI performance of nanoparticles was sought to be validated using the nude mouse model. A tumor-mimicking medical coupling gel phantom was established by the subcutaneous injection of medical coupling gel with different concentrations of PDFA nanoparticles. Dual-modal PAI and ultrasound imaging of lifelike tumor phantom cross-sections were conducted with our MSPAM system. Ultrasound imaging exhibited the contour profile of the tumor phantom, while the photoacoustic signal of PD-FA nanoparticle accumulation was captured to locate the tumor entity. As shown in Figure 6A, before PD-FA nanoparticle injection, no detectable photoacoustic signal could be captured in the tumor phantom. Then, a weak photoacoustic signal appeared after injection of $20 \mu \mathrm{g} \mathrm{mL} \mathrm{m}^{-1}$ PD-FA nanoparticles and increased with the concentration of PD-FA nanoparticles; the tumor phantom displayed a remarkable photoacoustic signal with $80 \mu \mathrm{g} \mathrm{mL}^{-1}$ PD-FA nanoparticles. By quantitatively analyzing the photoacoustic signal of the tumor phantom at different concentrations of PD-FA nanoparticles in Figure 6B, the signal intensity was well correlated with the concentration, demonstrating the possible quantification of the photoacoustic intensity of nanoparticles in vivo. Moreover, the tumor could be observed by PAI when the concentration of PD-FA nanoparticles was very low $\left(20 \mu \mathrm{g} \mathrm{mL}^{-1}\right)$, indicating the high sensitivity of PD-FA nanoparticles. These results showed the advantages of PAI with the aid of PD-FA nanoparticles.

Motivated by the remarkable PAI capability as conducted above, in vivo PAI was performed on the HeLa tumor-bearing nude mouse model. The photoacoustic images were obtained by our MSPAM system after intravenous injection of PD nanoparticles or PD-FA nanoparticles at different time intervals. Focusing our attention on Figure 7A, after injection of PD-FA nanoparticles, a photoacoustic signal was observed at 2 hours and became stronger with time until 24 hours. After that, the signal quickly decreased, which indicated that the PD-FA nanoparticles possessed the ability to target HeLa cells and accumulate in the tumor site, after which they were excreted from the mice. In contrast, a weak photoacoustic signal appeared at 8 hours and disappeared 12 hours after the intravenous injection of PD nanoparticles. This phenomenon implied a relatively long time for the PD-FA nanoparticles to take effect in the tumor. The distinction between the PD-FA nanoparticles and PD nanoparticles may be ascribed to the different mechanisms of nanoparticle aggregation: the PD-FA nanoparticles accumulated in the tumor site replied to donor-receptor interaction, while the PD nanoparticles targeted in tumor by the EPR (enhanced permeability and retention) effect existed in many other types of tumors. ${ }^{57-59}$ Quantitatively, in Figure 7B, the photoacoustic signal intensity after injection of PDFA nanoparticles was stronger than that of PD nanoparticle-
A
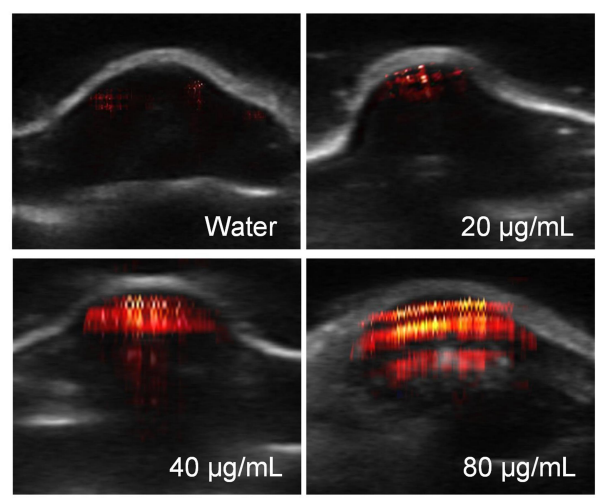

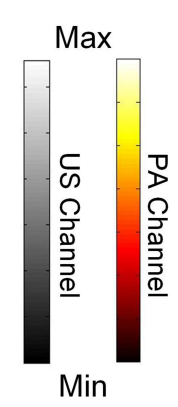

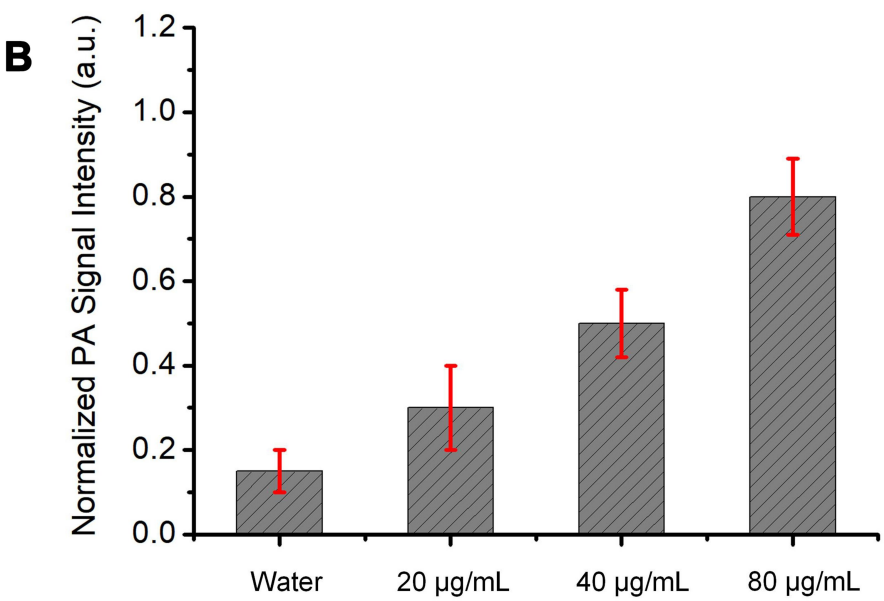

Figure 6 PAI in tumor-mimicking phantom. (A) Dual-modal PAI and ultrasound imaging of medical coupling gel with different concentration of PD-FA nanoparticles. (B) Quantization of the photoacoustic signal of tumor phantom with different concentration of PD-FA nanoparticles in (A). 
treated mice at all time points. In addition, the photoacoustic intensity of tumor with PD-FA nanoparticles was about five times larger than the tumor with PD nanoparticles at 24 hours post intravenous injection. As shown in Figure $7 \mathrm{C}$, we obtained a group of representative $3 \mathrm{D}$ photoacoustic images after intravenous injection nanoparticles at $8 \mathrm{~h}$. The shape, size and position of tumor could be clearly revealed in 3D images, and the photoacoustic signal intensity of tumors with PD-FA nanoparticles was obviously higher than that of tumors with PD nanoparticles. Besides, the
A

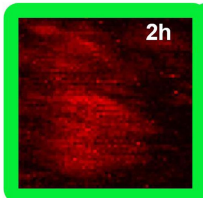

$12 \mathrm{~h}$
Targeted
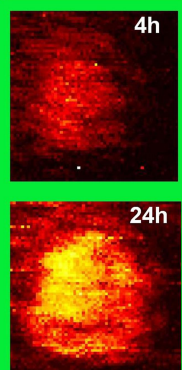

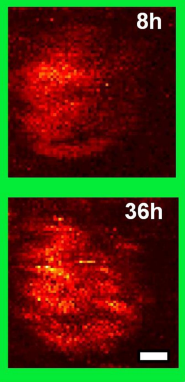

Nontargeted

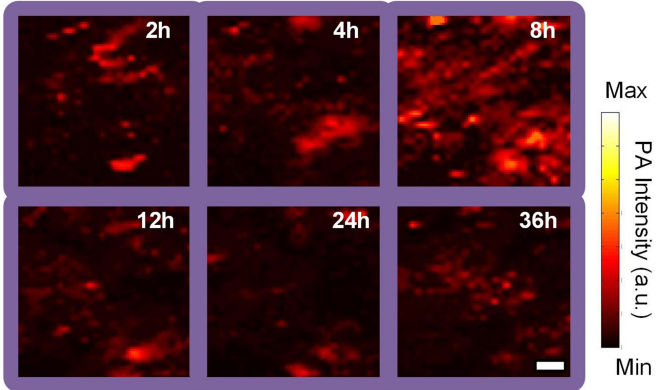

B
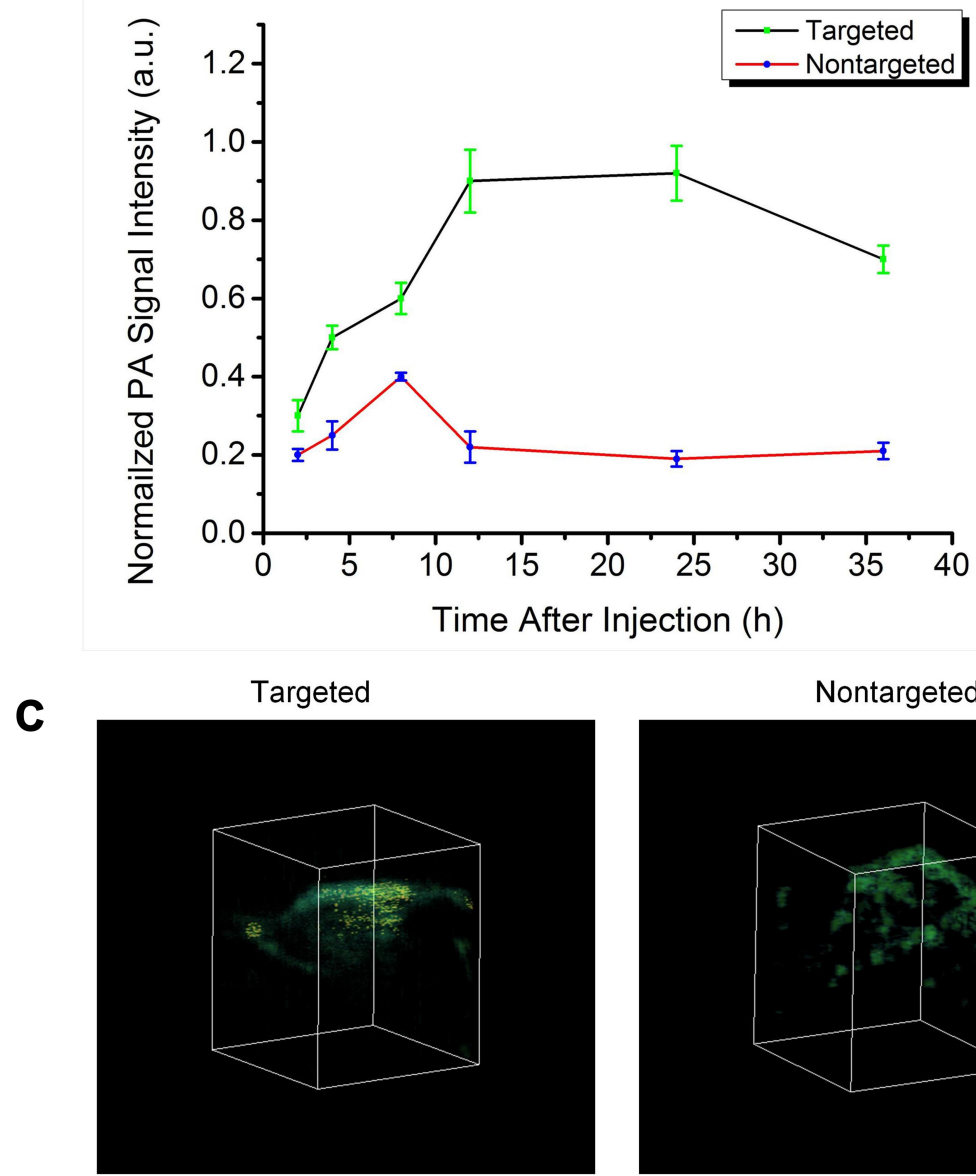

Nontargeted

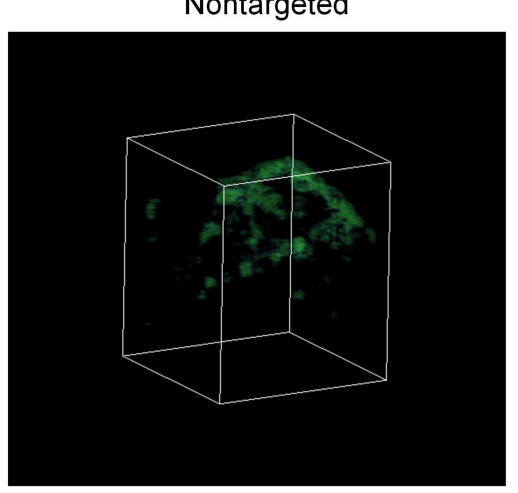

Min

Max

PA Intensity (a.u.)

Figure 7 In vivo PAI of PD-FA nanoparticles. (A) Photoacoustic images of tumor site in HeLa tumor-bearing nude mice after intravenous injection of PD nanoparticles or PD-FA nanoparticles for 2, 4, 8, I2, 24 and $36 \mathrm{~h}$. Scale bar is I mm. (B) Photoacoustic intensities of the tumor site at different time points obtained from (A). (C) In vivo 3D photoacoustic images of tumor tissues with injection of PD nanoparticles or PD-FA nanoparticles at $8 \mathrm{~h}$. 
outline of tumor with injection of PD-FA nanoparticles was clear than that with injection of PD nanoparticles. We also analyzed the raw data and obtained the quantitative photoacoustic signal in regions of tumor and surrounded normal tissue, which has been exhibited in Figure S9. The PAI intensity of tumor region after injection of PD-FA nanoparticles was about six times that of normal tissue, while the PAI intensity of tumor region after injection of PD nanoparticles was just approximately twice that of normal tissue. Taken together, the data indicate that the PD-FA nanoparticles possess excellent tumor targeting accumulation in $\mathrm{HeLa}$ tumor tissue, presenting gratifying PAI effects and a longlasting imaging period. In vivo multidimensional photoacoustic imaging has the ability of comprehensive imaging of cervical carcinoma.

\section{In vivo Photothermal Therapy Based on PD-FA Nanoparticles}

Inspired by the admirable efficacy of PTT in vitro and the excellent tumor targeting accumulation of PD-FA nanoparticles in HeLa cells, the in vivo PTT capability of PD-FA nanoparticles was investigated based on its exciting photoacoustic performance. This experiment was carried out on subcutaneous HeLa tumor-bearing nude mice; the mice in which the tumor size reached approximately $100 \mathrm{~mm}^{3}$ were selected and divided randomly into five groups $(\mathrm{n}=$ 6 per group) as follows: (1) PD-FA nanoparticles +845 nm (laser density: $1 \mathrm{~W} \mathrm{~cm}^{-2}$; irradiation time: 5 minutes); (2) PD-FA nanoparticles $+845 \mathrm{~nm}$; (3) PD-FA nanoparticles only; (4) Laser only; (5) Control.

According to the PAI results, the HeLa cell tumor exhibited the strongest photoacoustic signal after 24 hours post-injection. Then, PTT in tumor-bearing nude mice was conducted at 24 hours post-injection. In the first step, the photothermal effect of the nanoparticles in vivo was assessed by monitoring the temperature variation utilizing the infrared thermography. As shown in Figure $8 \mathrm{~A}$, the temperature at tumor site upon injection of PD-FA nanoparticles under laser therapy (laser density: $1 \mathrm{~W} \mathrm{~cm}^{-2}$ ) was gradually increased and reached up to $65^{\circ}$ $\mathrm{C}$ in 5 minutes, whereas the temperature at the tumor site upon injection of PD nanoparticles or PBS was just reach to $53^{\circ} \mathrm{C}$ or $38^{\circ} \mathrm{C}$. This result suggested that the PD-FA nanoparticles can generate hyperthermia in the tumor region of tumor-bearing nude mice. Quantitative photoacoustic signals in tumor site were recorded in Figure S10. Afterwards, to intuitively monitor the process of PTT and accurately record long-term PTT efficacy, the representative photograph and tumor volume of experimental mice were recorded every 3 days after different treatments. As revealed in Figure $8 \mathrm{~B}$, the tumors of mice in the single factor treatment groups (including PD-FA nanoparticle injection and free laser irradiation) were gradually growing at the same rate as the control group that was under no treatment, suggesting that neither the PD-FA nanoparticles alone nor the laser alone had any effect on tumor growth. The tumors treated by PD and PD-FA injection under laser irradiation exhibited excellent tumor ablation effect at the previous treatment stage. Unexpectedly, recurrence was observed in 18 days in the tumors treated by PD injection plus laser irradiation, which was marked with a red arrow in Figure 8B. The tumors treated by PD-FA injection plus laser irradiation completely removed with no recurrence, suggesting that the PD-FA nanoparticles have good targeting treatment ability in vivo. As presented in Figure $8 \mathrm{C}$, the relative tumor volume after 18 days of growth was about eight times larger than the initial tumor volume in the three groups. In contrast, the mice in the group with post-injection of nanoparticles under laser irradiation exhibited an absolutely different treatment effect. We carefully watched the mice under laser irradiation to ensure the efficacy and biosafety of the PTT, and the process of PTT was recorded in Figure S11. The surface of the tumor was slightly red and swollen after laser irradiation at 24 hours post-injection of the PD-FA nanoparticles. Then, black scabs formed after approximately one day and enlarged to become as large as the tumor surface in two days. As time went by, the black scab decreased, became smaller and was completely eradicated in the 18th day. These results indicated that PTT was capable of sufficiently damaging the tumors. Moreover, no obvious body weight variations in the mice were found among the five groups with different treatments (Figure 8D). This demonstrated that there were nearly no side effects of the PTT treatment process. Furthermore, we calculated survival ratio of the five groups after 30 days to evaluate the safety and efficiency of PTT. In Figure 8E, the subcutaneous tumorbearing mice in the groups of single factor treatment and the control group started to die during treatment process and exhibited low fraction surviving after 30 days. On the contrary, the mice in groups treated with PD-FA nanoparticles plus laser irradiation exhibited a $100 \%$ survival after 30 days. Finally, hematoxylin and eosin (H\&E) staining analyses of tumor tissue from different groups were conducted. As shown in Figure 8F, the groups of single factor 
A

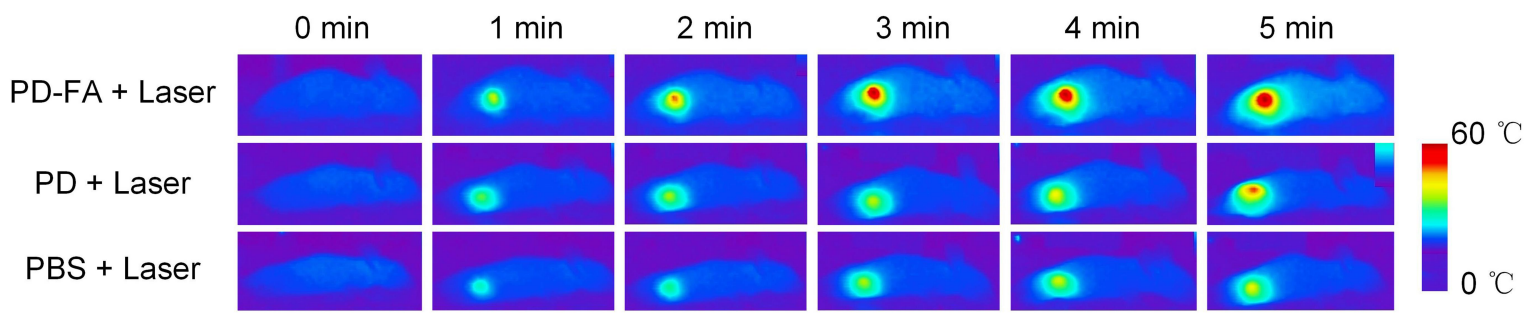

B

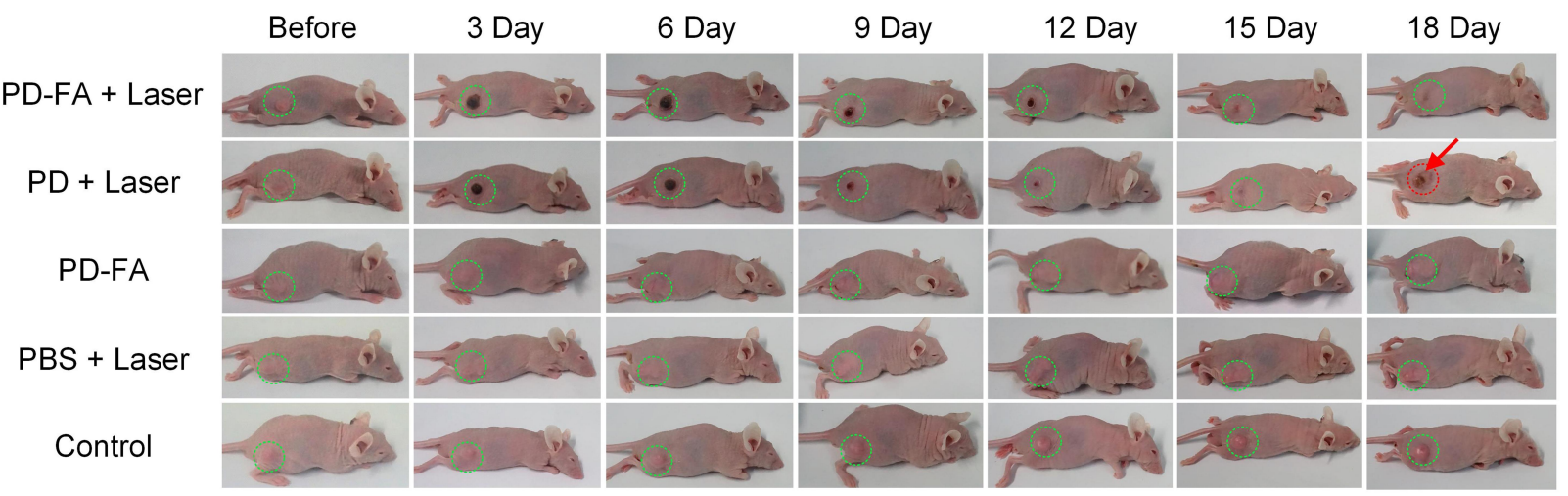

C

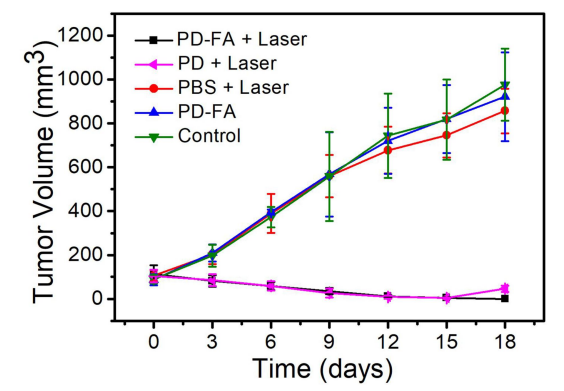

D

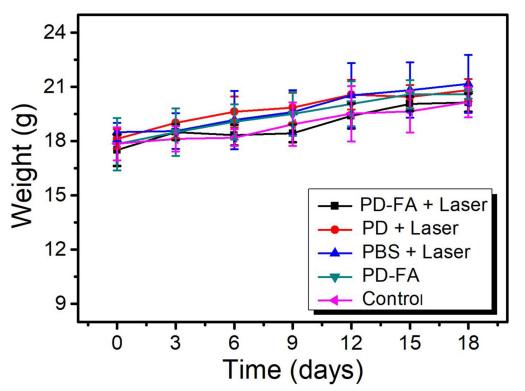

E

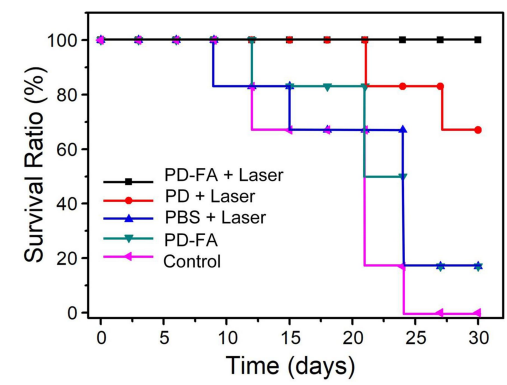

F
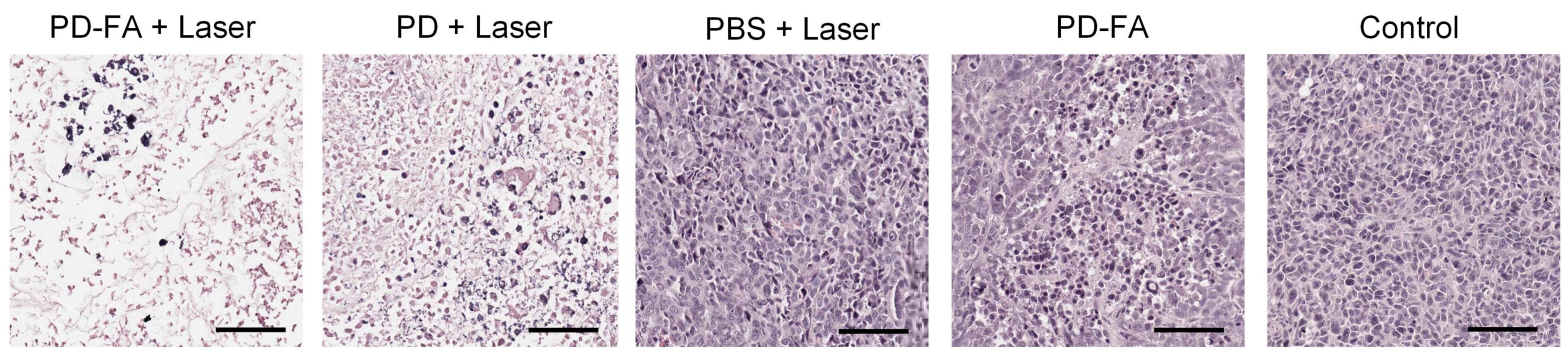

Figure 8 In vivo PTT of Hela tumor-bearing nude mice. (A) Thermal graphic images of HeLa tumor-bearing nude mice exposed to NIR laser for 5 min ( 845 nm, I W $\mathrm{cm}^{-2}$ ) after injection of PD-FA nanoparticles, PD nanoparticles or PBS. (B) Representative photos of HeLa tumor-bearing mice at different days after different treatments. Green circle indicated the tumor sites, red circle and red arrow indicated the recrudescence of tumor. (C) Tumor volume changes of mice after different treatments. (D) Body weight recording of mice after different treatment. (E) Survival rates of mice under different treatment after 30 days. (F) H\&E staining images of tumor sections collected from HeLa tumor-bearing mice with different treatments. The scale bar is $200 \mu \mathrm{m}$.

treatment and the control group showed no effect on the tumor cell, while the nanoparticles plus laser irradiation group exhibited damage to the tumor tissues through cell necrosis. Above results confirmed that the as-obtained PDFA nanoparticles were excellent photothermal agents with excellent targeting accumulation ability toward Hela cells for destroying tumor tissues in vivo, which was consistent with the in vitro results.

\section{Histological Analysis}

Last but not least, the biosafety of the biological reagent plays a crucial role for its potential clinical application. Then, the H\&E analysis of major organs including the heart, liver, spleen, lung, and kidneys after the different treatments discussed above was conducted. The results revealed in Figure 9 that there was no obvious injury as seen in the pathological image, which indicated that there 


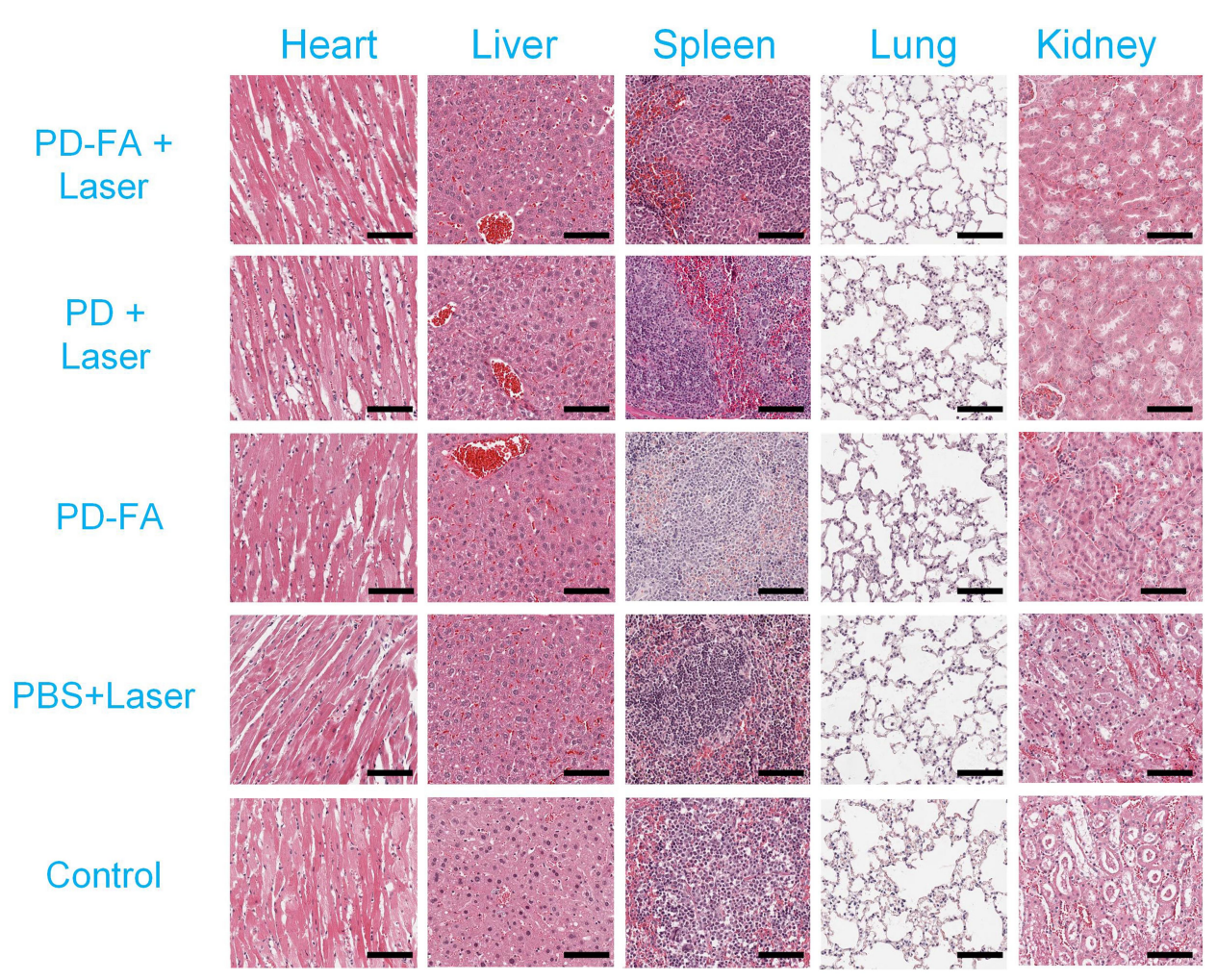

Figure 9 H\&E staining of major organs, including heart, liver, spleen, lung, and kidney of mice from each group after different treatment. The scale bar is $200 \mu \mathrm{m}$.

were no side effects of the PAI-guided PTT therapy with the PD-FA nanoparticles.

\section{Conclusions}

To summarize, PAI-guided PTT by aid of PD-FA nanoparticles as therapeutic agents is a promising treatment method for cervical cancer. As a contrast agent, the obtained PD-FA nanoparticles showed proper particle size, excellent biocompatibility and high absorption in the NIR window. Afterwards, PD-FA nanoparticles simultaneously exhibited strong photoacoustic signals and high photothermal conversion efficiency of up to $62.6 \%$. Based on the excellent Hela tumor targetability of the nanoparticles, PAI and photothermal tumor therapy in tumorbearing nude mice were conducted and demonstrated gratifying results. In vivo multi-scale PAI in cervical cancer showed clear identification of tumor location and shape, providing a potential tool to guide PTT that selective destruct the cervical cancer cell and no recurrence. As stated above, this method is in line with the principles of accurate diagnosis, accurate monitoring, and accurate treatment, which is expected to broaden the field of vision for the diagnosis and treatment of cervical cancer and promote the tumor therapy of other kinds of cancer.

\section{Acknowledgments}

This research is supported by the National Natural Science Foundation of China (11704082, 51903062 and 61905043), Startup grant from Guangzhou Medical University (B195002002019), Guangdong Basic and Applied Basic Research Foundation (2020A1515011104). The researchers also acknowledge the help of Wenyan Huang and Sujuan Zeng from Affiliated Stomatology Hospital of Guangzhou Medical University who contributed to revising the article and putting forward valuable suggestions.

\section{Disclosure}

The authors reported no conflicts of interest for this work.

\section{References}

1. Burk RD, Chen Z, Saller C, et al. Integrated genomic and molecular characterization of cervical cancer. Nature. 2017;543(7645):378-384. doi:10.1038/nature21386

2. Arbyn M, Castellsague X, de Sanjose S, et al. Worldwide burden of cervical cancer in 2008. Ann Oncol. 2011;22(12):2675-2686. doi:10.1093/annonc/mdr015

3. Ojesina AI, Lichtenstein L, Freeman SS, et al. Landscape of genomic alterations in cervical carcinomas. Nature. 2014;506(7488):371-375. doi:10.1038/nature12881 
4. Forman D, de Martel C, Lacey CJ, et al. Global burden of human papillomavirus and related diseases. Vaccine. 2012;30:F12-F23. doi:10.1016/j.vaccine.2012.07.055

5. Frisch M, Biggar RJ, Goedert JJ. Human papillomavirus-associated cancers in patients with human immunodeficiency virus infection and acquired immunodeficiency syndrome. J Natl Cancer. 2000;92 (18):1500-1510. doi:10.1093/jnci/92.18.1500

6. Wang R, Pan W, Jin L, et al. Human papillomavirus vaccine against cervical cancer: opportunity and challenge. Cancer Lett. 2020;471:88-102. doi:10.1016/j.canlet.2019.11.039

7. Wu ES, Urban RR, Krantz EM, et al. The association between HIV infection and cervical cancer presentation and survival in Uganda. Gynecol Oncol Rep. 2020;31:100516. doi:10.1016/j. gore.2019.100516

8. Yao J, Xia J, Wang LV. Multiscale functional and molecular photoacoustic tomography. Ultrason Imaging. 2016;38(1):44-62. doi: $10.1177 / 0161734615584312$

9. Liu Y, Nie L, Chen X. Photoacoustic molecular imaging: from multiscale biomedical applications towards early-stage theranostics. Trends Biotechnol. 2016;34(5):420-433. doi:10.1016/j. tibtech.2016.02.001

10. Qiu T, Lan YT, Gao WJ, et al. Photoacoustic imaging as a highly efficient and precise imaging strategy for the evaluation of brain disease. Quant Imaging Med Surg. 2020. doi:10.21037/qims-20-845

11. Lyu Y, Zeng J, Jiang Y, et al. Enhancing both biodegradability and efficacy of semiconducting polymer nanoparticles for photoacoustic imaging and photothermal therapy. ACS Nano. 2018;12 (2):1801-1810. doi:10.1021/acsnano.7b08616

12. Wang L, Xia J, Yao J, Maslov KI, Wang LV. Ultrasonically encoded photoacoustic flowgraphy in biological tissue. Phys Rev Lett. 2013;111(20):204301. doi:10.1103/PhysRevLett.111.204301

13. Zharov VP. Ultrasharp nonlinear photothermal and photoacoustic resonances and holes beyond the spectral limit. Nat Photonics. 2011;5(2):110-116. doi:10.1038/NPHOTON.2010.280

14. Xia Y, Xu C, Zhang X, et al. Liposome-based probes for molecular imaging: from basic research to the bedside. Nanoscale. 2019;11 (13):5822-5838. doi:10.1039/c9nr00207c

15. Zhang L, Sheng D, Wang D, et al. Bioinspired multifunctional melanin-based nanoliposome for photoacoustic/magnetic resonance imaging-guided efficient photothermal ablation of cancer. Theranostics. 2018;8(6):1591-1606. doi:10.7150/thno.22430

16. Chen J, Qi J, Chen C, et al. Tocilizumab-conjugated polymer nanoparticles for NIR-II photoacoustic-imaging-guided therapy of rheumatoid arthritis. Adv Mater. 2020;32(37):2003399. doi:10.1002/ adma.202003399

17. Chen J, Liu C, Hu D, et al. Single-layer MoS2 nanosheets with amplified photoacoustic effect for highly sensitive photoacoustic imaging of orthotopic brain tumors. Adv Funct Mater. 2016;26 (47):8715-8725. doi:10.1002/adfm.201603758

18. Wang D, Wu Y, Xia J. Review on photoacoustic imaging of the brain using nanoprobes. Neurophotonics. 2016;3(1):010901. doi:10.1117/1. NPh.3.1.010901

19. Strohm EM, Berndl ESL, Kolios MC. High frequency label-free photoacoustic microscopy of single cells. Photoacoustics. 2013;1(3-4):49-53. doi:10.1016/j.pacs.2013.08.003

20. Kim JY, Lee C, Park K, Lim G, Kim C. Fast optical-resolution photoacoustic microscopy using a 2-axis water-proofing MEMS scanner. Sci Rep. 2015;5(1):7932. doi:10.1038/srep07932

21. Cao R, Kilroy JP, Ning B, Wang T, Hossack JA, Hu S. Multispectral photoacoustic microscopy based on an optical-acoustic objective. Photoacoustics. 2015;3(2):55-59. doi:10.1016/j.pacs.2014.12.004

22. Guggenheim JA, Allen TJ, Plumb A, et al. Photoacoustic imaging of human lymph nodes with endogenous lipid and hemoglobin contrast. J Biomed Opt. 2015;20(5):1. doi:10.1117/1.JBO.20.5.050504
23. Cui C, Yang Z, Hu X, et al. Organic Semiconducting nanoparticles as efficient photoacoustic agents for lightening early thrombus and monitoring thrombolysis in living mice. ACS Nano. 2017;11 (3):3298-3310. doi:10.1021/acsnano.7b00594

24. Karande GY, Hedgire SS, Sanchez Y, et al. Advanced imaging in acute and chronic deep vein thrombosis. Cardiovasc Diagn Ther. 2016;6(6):493-507. doi:10.21037/cdt.2016.12.06

25. Wang $\mathrm{LV}, \mathrm{Hu} \mathrm{S}$. Photoacoustic tomography: in vivo imaging from organelles to organs. Science. 2012;335(6075):1458-1462. doi:10.1126/science. 1216210

26. Nie L, Cai X, Maslov K, Garcia-Uribe A, Anastasio MA, Wang LV. Photoacoustic tomography through a whole adult human skull with a photon recycler. J Biomed Opt. 2012;17(11):110506. doi:10.1117/1. JBO.17.11.110506

27. Xia J, Yao J, Wang LV. Photoacoustic tomography: principles and advances. Prog Electromagn Res. 2014;147:1-22. doi:10.2528/ pier14032303

28. Guo B, Chen J, Chen N, et al. High-resolution 3D NIR-II photoacoustic imaging of cerebral and tumor vasculatures using conjugated polymer nanoparticles as contrast agent. Adv Mater. 2019;31 (25):1808355. doi:10.1002/adma.201808355

29. Ren Y, Sedgwick AC, Chen J, et al. Manganese(II) texaphyrin: a paramagnetic photoacoustic contrast agent activated by Near-IR light. J Am Chem Soc. 2020;142(38):16156-16160. doi:10.1021/ jacs.0c04387

30. Sun J, Cai W, Sun Y, Guo C, Zhang R. Facile synthesis of melanin-dye nanoagent for NIR-II fluorescence/photoacoustic imaging-guided photothermal therapy. Int $J$ Nanomedicine. 2020;15:10199-10213. doi:10.2147/IJN.S284520

31. Fang X, Lui KH, Li S, et al. Multifunctional nanotheranostic gold nanocage/selenium core-shell for PAI-guided chemo-photothermal synergistic therapy in vivo. Int $J$ Nanomedicine. 2020;15:10271-10284. doi:10.2147/IJN.S275846

32. Bao Y-W, Hua X-W, Li Y-H, Jia H-R, Wu F-G. Hyperthemiapromoted cytosolic and nuclear delivery of copper/carbon quantum dot-crosslinked nanosheets: multimodal imaging-guided photothermal cancer therapy. ACS Appl Mater Interfaces. 2018;10 (2):1544-1555. doi:10.1021/acsami.7b15332

33. O’Neal DP, Hirsch LR, Halas NJ, Payne JD, West JL. Photo-thermal tumor ablation in mice using near infrared-absorbing nanoparticles. Cancer Lett. 2004;209(2):171-176. doi:10.1016/j.canlet.2004.02.004

34. Robinson JT, Tabakman SM, Liang Y, et al. Ultrasmall reduced graphene oxide with high near-infrared absorbance for photothermal therapy. J Am Chem Soc. 2011;133(17):6825-6831. doi:10.1021/ ja2010175

35. Zhao G, Wu H, Feng R, et al. Novel metal polyphenol framework for MR imaging-guided photothermal therapy. ACS Appl Mater Interfaces. 2018;10(4):3295-3304. doi:10.1021/acsami.7b16222

36. Gao D, Zhang B, Liu Y, et al. Molecular engineering of near-Infrared light-responsive BODIPY-based nanoparticles with enhanced photothermal and photoacoustic efficiencies for cancer theranostics. Theranostics. 2019;9(18):5315-5331. doi:10.7150/thno.34418

37. Shimoji Y, Nagai Y, Toita T, et al. A Phase II study of neoadjuvant chemotherapy followed by extended field concurrent chemoradiotherapy for para-aortic lymph node positive cervical cancer. Anticancer Res. 2020;40(6):3565-3570. doi:10.21873/anticanres.14346

38. Xiao Y, Cheng H, Wang L, Yu X. Clinical response and safety of apatinib monotherapy in recurrent, metastatic cervical cancer after failure of chemotherapy: a retrospective study. $J$ Gynecol Oncol. 2020;31(1):e2. doi:10.3802/jgo.2020.31.e2

39. Li H, Pang Y, Cheng X. Surgery of primary sites for stage IVB cervical cancer patients receiving chemoradiotherapy: a population-based study. J Gynecol Oncol. 2020;31(1):e8. doi:10.3802/jgo.2020.31.e8 
40. Cheng L, Wang C, Feng L, Yang K, Liu Z. Functional nanomaterials for phototherapies of cancer. Chem Rev. 2014;114(21):10869-10939. doi:10.1021/cr400532z

41. Phan TTV, Bui NQ, Cho S-W, et al. Photoacoustic imaging-guided photothermal therapy with tumor-targeting HA-FeOOH@PPy nanorods. Sci Rep. 2018;8(1):8809. doi:10.1038/s41598-018-27204-8

42. Cai Y, Si W, Tang Q, et al. Small-molecule diketopyrrolopyrrolebased therapeutic nanoparticles for photoacoustic imaging-guided photothermal therapy. Nano Res. 2017;10(3):794-801. doi:10.1007/ s12274-016-1332-2

43. Wang J, Yan R, Guo F, Yu M, Tan F, Li N. Targeted lipid-polyaniline hybrid nanoparticles for photoacoustic imaging guided photothermal therapy of cancer. Nanotechnology. 2016;27(28):285102. doi:10.1088/0957-4484/27/28/285102

44. Bao T, Yin W, Zheng X, et al. One-pot synthesis of PEGylated plasmonic MoO3-x hollow nanospheres for photoacoustic imaging guided chemo-photothermal combinational therapy of cancer. Biomaterials. 2016;76:11-24. doi:10.1016/j.biomaterials.2015.10.048

45. Guo W, Qiu Z, Guo C, et al. Multifunctional theranostic agent of $\mathrm{Cu}-2(\mathrm{OH}) \mathrm{PO} 4$ quantum dots for photoacoustic image-guided photothermal/photodynamic combination cancer therapy. ACS Appl Mater Interfaces. 2017;9(11):9348-9358. doi:10.1021/acsami.6b15703

46. Jun SW, Manivasagan P, Kwon J, et al. Folic acid-conjugated chitosan-functionalized graphene oxide for highly efficient photoacoustic imaging-guided tumor-targeted photothermal therapy. Int $\mathrm{J} \mathrm{Biol}$ Macromol. 2020;155:961-971. doi:10.1016/j.ijbiomac.2019.11.055

47. Bao X, Yuan Y, Chen J, et al. In vivo theranostics with near-infraredemitting carbon dots-highly efficient photothermal therapy based on passive targeting after intravenous administration. Light-Sci Appl. 2018;7. doi:10.1038/s41377-018-0090-1

48. Song J, Zhang L, Yi H, et al. NIR-responsive nanoplatform for pre/ intraoperative image-guided carcinoma surgery and photothermal ablation of residual tumor tissue. Nanomed Nanotechnol. 2019;20:102020. doi:10.1016/j.nano.2019.102020

49. Ditto AJ, Shah KN, Robishaw NK, Panzner MJ, Youngs WJ, Yun YH. The interactions between L-Tyrosine based nanoparticles decorated with folic acid and cervical cancer cells under physiological flow. Mol Pharm. 2012;9(11):3089-3098. doi:10.1021/mp300221f

50. Luong D, Kesharwani P, Alsaab HO, et al. Folic acid conjugated polymeric micelles loaded with a curcumin difluorinated analog for targeting cervical and ovarian cancers. Colloids Surf B Biointerfaces. 2017;157:490-502. doi:10.1016/j.colsurfb.2017.06.025
51. Lin J, Hu W, Gao F, Qin J, Peng C, Lu X. Folic acid-modified diatrizoic acid-linked dendrimer-entrapped gold nanoparticles enable targeted CT imaging of human cervical cancer. J Cancer. 2018;9 (3):564-577. doi:10.7150/jca. 19786

52. Wei Z, Wu M, Lan S, et al. Semiconducting polymer-based nanoparticles for photothermal therapy at the second near-infrared window. Chem Commun. 2018;54(96):13599-13602. doi:10.1039/c8cc07583b

53. Zhang J, Chen H, Zhou $\mathrm{T}$, et al. A PIID-DTBT based semi-conducting polymer dots with broad and strong optical absorption in the visible-light region: highly effective contrast agents for multiscale and multi-spectral photoacoustic imaging. Nano Res. 2017;10(1):64-76. doi:10.1007/s12274-016-1266-8

54. Wei Z, Xin F, Zhang J, et al. A donor-acceptor conjugated polymer-based nanoparticle for highly effective photoacoustic imaging and photothermal therapy in NIR-II window. Chem Commun. 2020;56:1093-1096. doi:10.1039/c9cc07821e

55. Avalos A, Haza AI, Mateo D, Morales P. Cytotoxicity and ROS production of manufactured silver nanoparticles of different sizes in hepatoma and leukemia cells. J Appl Toxicol. 2014;34(4):413-423. doi:10.1002/jat.2957

56. Jiang Y, Upputuri PK, Xie C, et al. Broadband absorbing semiconducting polymer nanoparticles for photoacoustic imaging in second near-infrared window. Nano Lett. 2017;17(8):4964-4969. doi:10.1021/acs.nanolett.7b02106

57. Tanaka T, Shiramoto S, Miyashita M, Fujishima Y, Kaneo Y. Tumor targeting based on the effect of enhanced permeability and retention (EPR) and the mechanism of receptor-mediated endocytosis (RME). Int J Pharm. 2004;277(1-2):39-61. doi:10.1016/j.ijpharm.2003.09.050

58. Kobayashi H, Watanabe R, Choyke PL. Improving conventional enhanced permeability and retention (EPR) effects; what is the appropriate target? Theranostics. 2014;4(1):81-89. doi:10.7150/thno.7193

59. Fan Z, Liu H, Xue Y, et al. Reversing cold tumors to hot: an immunoadjuvant-functionalized metal-organic framework for multimodal imaging-guided synergistic photo-immunotherapy. Bioact Mater. 2020;6(2):312-325. doi:10.1016/j.bioactmat.2020.08.005
International Journal of Nanomedicine

\section{Publish your work in this journal}

The International Journal of Nanomedicine is an international, peerreviewed journal focusing on the application of nanotechnology in diagnostics, therapeutics, and drug delivery systems throughout the biomedical field. This journal is indexed on PubMed Central, MedLine, CAS, SciSearch ${ }^{\mathbb{R}}$, Current Contents ${ }^{\mathbb{R}} /$ Clinical Medicine, $^{2}$

\section{Dovepress}

Journal Citation Reports/Science Edition, EMBase, Scopus and the Elsevier Bibliographic databases. The manuscript management system is completely online and includes a very quick and fair peer-review system, which is all easy to use. Visit http://www.dovepress.com/ testimonials.php to read real quotes from published authors. 